MODELING, IDENTIFICATION AND CONTROL, 2002, VOL. 23, NO. 1, 5-26

doi:10.4173/mic.2002.1.1

\title{
Fluid Flow Simulations of a Vane Separator*
}

\author{
AAGE I. JØSANG and MORTEN CHR. MELAAEN†
}

Keywords: CFD, vanes, measurement, verification, droplet-gas flow

In this work, the fluid flow in a vane separator is simulated by Computational Fluid Dynamics (CFD). Detailed measurements of air flow inside a single passage in a vane separator have earlier been presented (Jøsang and Melaaen, 2000). The simulations cover various turbulence models ( $k-\varepsilon$ and RSM turbulence models), near wall treatments, numerical schemes and structured grid as well as unstructured grid simulations. Earlier simulations show that a proper matching between the grid and the near wall treatment is important. However, most important is the effect of the two different numerical schemes together with the turbulence models. The selected numerical schemes are two different first order schemes (the power law scheme and the first order upwind scheme) and the higher order QUICK scheme. Normally, the highest order scheme is asssumed to produce the most accurate result, but in the present work the combination of the best turbulence model (RSM) and QUICK gave the worst result. Most of the other combinations gave good predicted flow fields, although some deviations exist for the prediction of the recirculation zones.

\section{Introduction}

The separation of droplets from a gas stream is a matter of necessity in many processes. The equipment used for this separation is widely spread from various types of cyclones, vane-type separators, packed bed separators, scrubbers, and wire/fibre separators. In this work a vane-type separator is studied. This type consists of several vanes side-by-side creating narrow passages, which the gas must pass. The inertial forces are the driving mechanism of the separation. The vanes are curved to force the gas to change direction, and the relative large inertia of the droplets causes the droplets to collide with the vane. This will lead to the formation of a liquid film, which must be drained. The design of the vane is crucial for the separation efficiency, and the vane may contain 'pockets' where the collected fluid can drain without influence from the passing gas. The passing gas can cause some of the already separated liquid to re-cntrain, and hence gas velocity is a limiting factor. If the gas velocity is too high some of the liquid, now existing at the wall as a film (continuously or non-continuously), may be sheared off the liquid film under the action of the shear stress from the gas, causing a decrease in the efficiency. Sharp corners and other wall-roughness induce a lower gas velocity for the re-entrainment to occur.

The motivation for this work is to increase the knowledge of the droplet-gas flow inside the narrow passage in a vane separator. One of the steps when increasing the knowledge is to do single phase simulations of the air flow in the vane separator, and this work is presented in this article. In the literature, previous work concerning the use of CFD on this type of process equipment is often limited to a study on the

*An early version of this article was presented at the SIMS 2001 conference, October 8th9th 2001, Porsgrunn, Norway.

$†$ Telemark University College (HiT-TF) and Telemark R\&D Centre (Tel-Tek), Kjølnes Ring 56, 3917 Porsgrunn. Norway. E-mail: Morten.C.Melaaen@hit.no 
efficiency of the separator (Wang \& James, 1998 and Wang \& Davies, 1996). Verlaan (1991) presents one measured profile along with predicted values from a CFD code, in addition to analysis considering the efficiency of the separator and distribution of the film in the separator. Verlaan also uses the CFD code directly in the design of a new vane. Keshava Iyer et al., 1997 present some measured velocity profiles in a chevron packet, but no CFD is used in this reference. Other references present measurements and/or analytical work to describe the efficiency of such separators, but none of these references contains measurements of the gas flow within the separator and comparisons with predicted values from a CFD code (Nakao et al., 1999, Kudo et al., 1996 and Monat et al., 1986).

The model, in which the experiments are carried out, has been simulated by the use of a CFD code. The model is simulated as it appears in the experiment, to ensure the same basis for the comparisons. The CFD code used is Fluent, and both the structured and unstructured versions are used, Fluent v4.5 and Fluent v5.3, respectively. The appropriate grid is generated in Gambit, and certain grid considerations must be fulfilled. First the grid has to be fine enough to resolve the flow details. In addition, the near wall treatment for the turbulence leads to certain constraints on the grid. The presence of the walls will influence the flow, and the near wall modelling of the turbulence is important. The two turbulence models, the $k-\varepsilon$ turbulence model and the Reynolds Stress Model (RSM), are tested and compared. This analysis is also done both when first order scheme and higher order scheme (QUICK) are used.

\section{Experimental model of the vane separator}

The model of the vane separator unit is designed to give a good basis for the measurements (Figure 1). The fluid flow in the experimental model is studied by Laser Doppler Anemometry (LDA). Detailed velocity profiles appear and mean velocities and turbulence properties can be calculated. Figure 1 shows the physical model. The centre distance between the vanes is $2.5 \mathrm{~cm}$. More details about these measurements are given by Jøsang and Melaaen (2000).

The positions measured can be identified in Figure 2. In addition, an inlet profile is measured $50 \mathrm{~mm}$ upstream the vanes, that is $\mathrm{x}=-50 \mathrm{~mm}$. In this position a fully developed flow is measured. The comparison between simulations and measurements are done in the same positions as showed in Figure $2($ not in $\mathrm{x}=0.0 \mathrm{~mm})$.

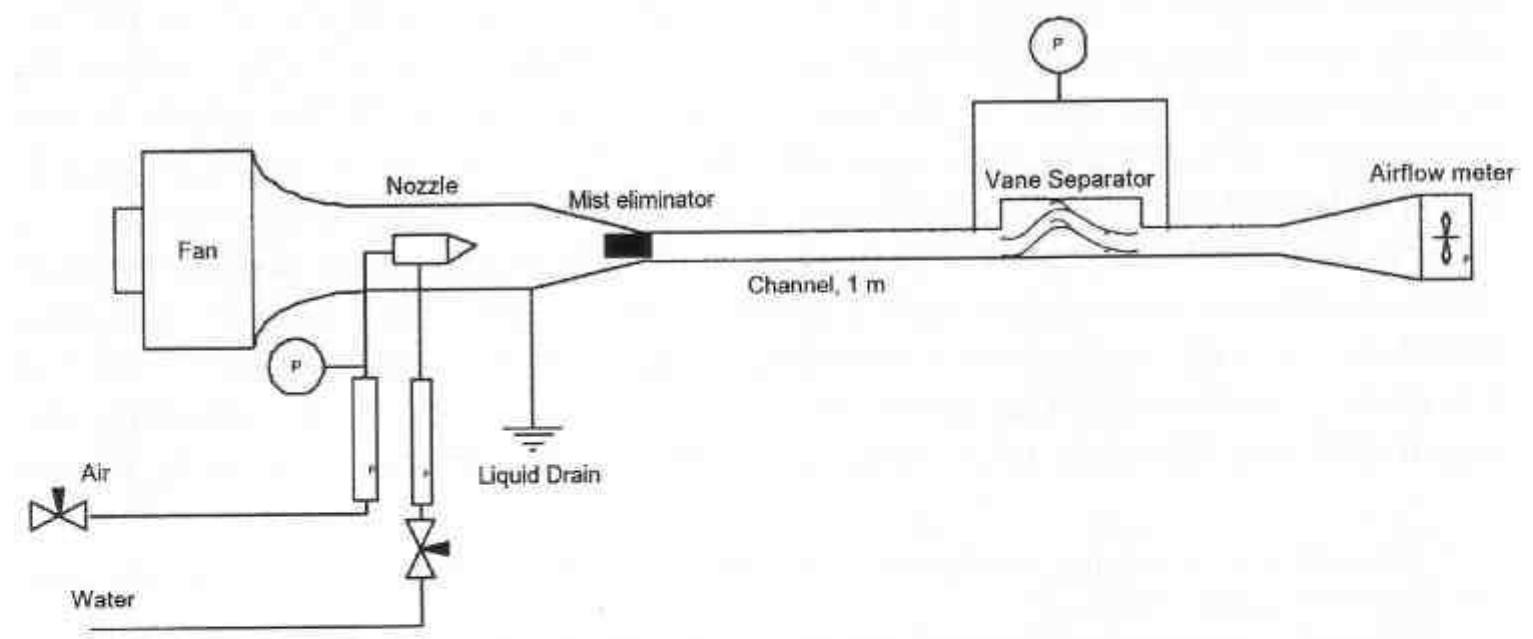

Figure 1. Principal sketch over the experimental model. 


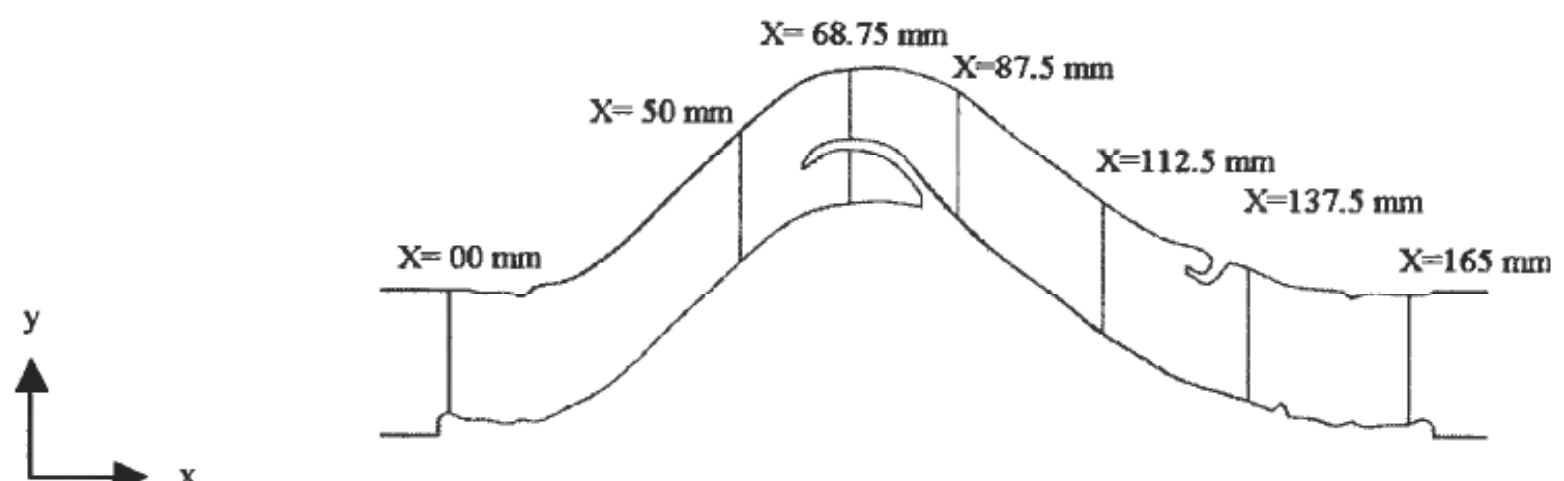

Figure 2. The different positions of the measured profiles in the vane separator.

\section{Conservation equations}

The fluid flow inside the vane separator is turbulent. In this work, both the $k$ - $\varepsilon$ turbulence model and the Reynolds Stress Model (RSM) have been used, and hence the equations presented below are shown both for the $k-\varepsilon$ turbulence model and the RSM model. The equations for steady flow written in Cartesian tensor notation are shown.

\section{Continuity equation}

$$
\frac{\partial}{\partial x_{j}}\left(\rho u_{j}\right)=0
$$

Momentum equation

$$
\frac{\partial}{\partial x_{j}}\left(\rho u_{i} u_{j}\right)=\frac{\partial}{\partial x_{j}}\left(\sigma_{i j}\right)-\frac{\partial}{\partial x_{j}}\left(\rho \overline{u_{i}^{\prime} u_{j}^{\prime}}\right)
$$

where the stress tensor, $\sigma_{i j}$, for laminar flow can be divided into a pressure part and a shear stress part and is given by

$$
\sigma_{i j}=-p \delta_{i j}+\tau_{i j}=-\left(p+\frac{2}{3} \mu \frac{\partial u_{l}}{\partial x_{l}}\right) \delta_{i j}+\mu\left(\frac{\partial u_{i}}{\partial x_{j}}+\frac{\partial u_{j}}{\partial x_{i}}\right)
$$

The velocities, $u_{i}$, are the mean velocities, while the velocity fluctuations, $u_{i}^{\prime}$, are due to turbulence. When the momentum equation is averaged, the Reynolds stresses $\left(\rho u_{i}^{\prime} u_{j}^{\prime}\right)$ in Equation 2 appear and these stresses need additional models since they are new variables in the system. The next step is to present the turbulence models needed for calculating the Reynolds stresses. The selected models are the $k-\varepsilon$ turbulence model and the Reynolds Stress Model (RSM).

\section{$k-\varepsilon$ turbulence model}

In the $k-\varepsilon$ turbulence model, the Reynolds stresses are given by

$$
-\rho \overline{u_{i}^{\prime} u_{j}^{\prime}}=-\frac{2}{3}\left(\rho k+\mu_{t} \frac{\partial u_{l}}{\partial x_{l}}\right) \delta_{i j}+\mu_{t}\left(\frac{\partial u_{i}}{\partial x_{j}}+\frac{\partial u_{j}}{\partial x_{i}}\right)
$$


The turbulent viscosity can be written

$$
\mu_{t}=\rho C_{\mu} \frac{k^{2}}{\varepsilon}
$$

where $k$ is the turbulent kinetic energy and $\varepsilon$ the dissipation rate of turbulent kinetic energy. The conservation equation for turbulent kinetic energy is

$$
\frac{\partial}{\partial x_{j}}\left(\rho u_{j} k\right)=\frac{\partial}{\partial x_{j}}\left[\frac{\mu_{t}}{\sigma_{k}} \frac{\partial k}{\partial k_{j}}\right]+G-\varepsilon \rho
$$

The conservation equation for the dissipation rate of turbulent kinetic energy is

$$
\frac{\partial}{\partial x_{j}}\left(\rho u_{j} \varepsilon\right)=\frac{\partial}{\partial x_{j}}\left[\frac{\mu_{t}}{\sigma_{\varepsilon}} \frac{\partial \varepsilon}{\partial k_{j}}\right]+C_{1} \frac{\varepsilon}{k} G-C_{2} \frac{\varepsilon^{2}}{k} \rho
$$

where $G$ is the production rate and is given as

$$
G=\mu_{t}\left(\frac{\partial u_{i}}{\partial x_{i}}+\frac{\partial u_{i}}{\partial x_{j}}\right) \frac{\partial u_{j}}{\partial x_{i}}
$$

Standard coefficients are selected in the $k-\varepsilon$ turbulence model and shown in Table 1.

Table 1. The constants in the $k-\varepsilon$ turbulence model

\begin{tabular}{ccccc}
\hline$C_{1}$ & $C_{2}$ & $C_{\mu}$ & $\sigma_{k}$ & $\sigma_{\varepsilon}$ \\
\hline 1.44 & 1.92 & 0.09 & 1.0 & 1.3 \\
\hline
\end{tabular}

\section{Reynolds Stress Model (RSM)}

In the Reynolds Stress Model (RSM), the Reynolds stresses, $\rho \overline{u_{i}^{\prime} u_{j}^{\prime}}$, are found from $\overline{u_{i}^{\prime} u_{j}^{\prime}}$, which are given by conservation equations. This model takes into account the anisotropy of the turbulence. The conservation equations can be written (Fluent, 1996)

$$
\frac{\partial}{\partial x_{k}}\left(\rho u_{k} \overline{u_{i}^{\prime} u_{j}^{\prime}}\right)=D_{i j}+P_{i j}+\Phi_{i j}^{\prime}-\varepsilon_{i j}
$$

On the left hand side the term gives the convection of $\overline{u_{i}^{\prime} u_{j}^{\prime}}$, and the first term on right hand side is the diffusion term, $D_{i j}$. The production term, $P_{i j}$, follows. The two last terms are the pressure-strain redistribution, $\Phi_{i j}$, and the dissipation rate, $\varepsilon_{i j}$. These terms can from equation manipulation of the momentum equation exactly be written like

$$
\begin{gathered}
D_{i j}=-\frac{\partial}{\partial x_{k}}\left[\left(\rho \overline{u_{i}^{\prime} \overline{u_{j}^{\prime} u_{k}^{\prime}}}\right)+\overline{p^{\prime}\left(\delta_{k j} u_{i}^{\prime}+\overline{\delta_{i k} u_{j}^{\prime}}\right)}-\mu \frac{\partial}{\partial x_{k}}\left(\overline{u_{i}^{\prime} u_{j}^{\prime}}\right)\right] \\
P_{i j}=-\rho \overline{u_{i}^{\prime} u_{k}^{\prime}} \frac{\partial u_{j}}{\partial x_{k}}-\rho \overline{u_{j}^{\prime} u_{k}^{\prime}} \frac{\partial u_{i}}{\partial x_{k}} \\
\Phi_{i j}=\overline{p^{\prime}\left[\frac{\partial u_{i}^{\prime}}{\partial x_{j}}+\frac{\partial u_{j}^{\prime}}{\partial x_{i}}\right]}
\end{gathered}
$$




$$
\varepsilon_{i j}=2 \mu\left[\overline{\left[\frac{\partial u_{i}^{\prime}}{\partial x_{k}}+\frac{\partial u_{j}^{\prime}}{\partial x_{k}}\right]}\right.
$$

Based on the conservation equation for $\overline{u_{i}^{\prime} u_{j}^{\prime}}$, it is possible to find $\overline{u_{i}^{\prime} u_{j}^{\prime}}$. However, it is more unknown variables than equations. This is the closure problem. New models must be included so that it is the same number of variables and equations. If the production term, $P_{i j}$, is studied, it appears that this term can be calculated directly since both $u_{i}$ and $\overline{u_{i}^{\prime} u_{j}^{\prime}}$ already have conservation equations. However, the other three terms need models $\left(D_{i j}, \Phi_{i j}\right.$ and $\left.\varepsilon_{i j}\right)$. A simple model for the diffusion term is

$$
D_{i j}=\frac{\partial}{\partial x_{k}}\left(\frac{\mu_{i}}{\sigma_{k}} \frac{\partial\left(\overline{u_{i}^{\prime} u_{j}^{\prime}}\right)}{\partial x_{k}}\right)
$$

The pressure-strain redistribution, $\Phi_{i j}$, is modelled by

$$
\Phi_{i j}=-C_{1} \frac{\varepsilon \rho}{k}\left[\overline{u_{i}^{\prime} u_{j}^{\prime}}-\frac{2}{3} \delta_{i j} k\right]-C_{2}\left[P_{i j}-\frac{2}{3} \delta_{i j} P-\frac{\rho}{2}\left(\frac{\partial u_{j}}{\partial x_{i}}+\frac{\partial u_{i}}{\partial x_{j}}\right)\right]
$$

where $P=P_{i i} / 2$. The dissipation rate, $\varepsilon_{i j}$, is modelled by

$$
\varepsilon_{i j}=\frac{2}{3} \delta_{i j} \varepsilon
$$

$\varepsilon$ is not known and must be found. This can be done by solving a conservation equation for $\varepsilon$ similar to the one used in the $k-\varepsilon$ turbulence model (Equation 7). The production term $G$ is given by $G=P_{i i} / 2$. When the RSM model is selected instead of the $k-\varepsilon$ turbulence model, the $k$-equation will not be solved, but instead equations for the cross moments, $\overline{u_{i}^{\prime} u_{j}^{\prime}}$, are solved. The constants used in the RSM model are shown in Table 2.

Table 2. The constants in the RSM turbulence model

\begin{tabular}{lll}
\hline$C_{1}$ & $C_{2}$ & $\sigma_{k}$ \\
\hline 1.8 & 0.6 & 1.0
\end{tabular}

\section{Scalar conservation equation}

The continuity, momentum and turbulence equations can be treated partly as a scalar equation of the form

$$
\frac{\partial}{\partial x_{j}}\left(\rho u_{j} \varphi\right)=\frac{\partial}{\partial x_{j}}\left(\Gamma \frac{\partial \varphi}{\partial x_{j}}\right)+S
$$

and $\varphi, \Gamma$ and $S$ are the dependent variable, the diffusion coefficient and the source term, respectively. The terms from left to right is the convective, diffusive and source terms. When the models are discretised, this equation will be analysed.

\section{Finite volume discretisation}

In this article, both a structured and an unstructured grid are used in the analysis of the fluid flow. The finite volume discretisation shown here is done for a structured 


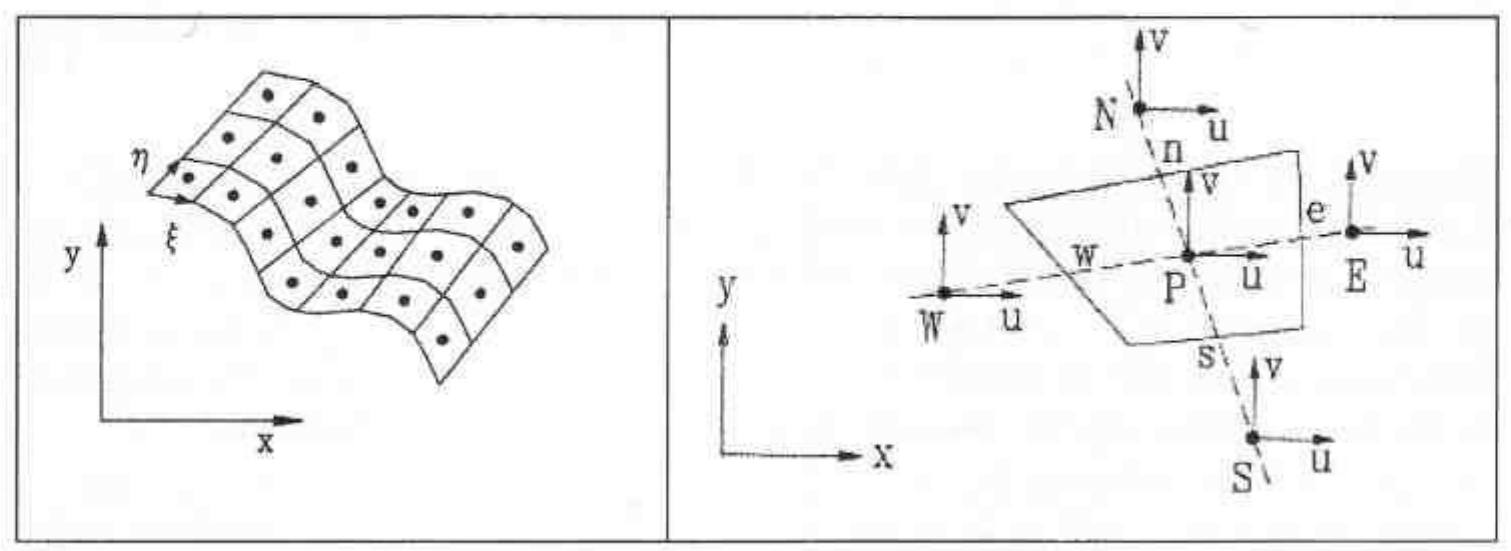

Figure 3. Left: Curvilinear non-orthogonal coordinates. Right: General two-dimensional control volume with velocities and scalar variables saved in the centre.

grid developed by curvilinear non-orthogonal coordinates shown in Figure 3. For unstructured grid it will be more complex, and will not be shown.

\section{Integration of the conservation equation}

The general scalar conservation equation on a coordinate invariant, strong conservation form is written

$$
\nabla \cdot \vec{J}=S \quad \vec{J}=\rho \vec{U} \varphi-\Gamma \nabla \varphi
$$

They are redefined for the different conservation equations that are solved. Since the finite-volume concept is chosen, the equation is integrated over a general control volume, $\delta V$, around grid point $P$ in physical space (Figure 3 ) and then the divergence theorem is used

$$
\left.\vec{J} \cdot \vec{A}^{(1)}\right|_{e}-\left.\vec{J} \cdot \vec{A}^{(1)}\right|_{w}+\left.\vec{J} \cdot \vec{A}^{(2)}\right|_{n}-\left.\vec{J} \cdot \vec{A}^{(2)}\right|_{s}=\bar{S}_{P}
$$

where

$$
\left.\vec{J} \cdot \vec{A}^{(i)}\right|_{n n} \int_{A_{n n}} \vec{J} \cdot d \vec{A}^{(i)}=\left(\rho \vec{U} \cdot \vec{A}^{(i)} \varphi-\Gamma \vec{A}^{(i)} \cdot \nabla \varphi\right)_{n n}=\hat{J}_{n n}^{i}
$$

and the linearised source term can be written as

$$
\bar{S}_{P}=\int_{\delta V} S d V=\bar{S}_{1 P}+\bar{S}_{2 P} \varphi_{P}
$$

where the subscripts $e, w, n$ and $s$ indicate the different faces of the control volume, and $n n$ is equal to $e$ or $w$ when $i=1$ and $n n$ is equal to $n$ or $s$ when $i=2$.

\section{Coordinate transformations}

The integrated equation is transformed from Cartesian coordinates (physical domain, $\left.\left(x_{i}\right)=(x, y)\right)$, to curvilinear non-orthogonal coordinates (computational domain, $\left.\left(\xi^{i}\right)=(\xi, \eta)\right)$, by the chain rule (Figure 3 ). 


$$
\frac{\partial \varphi}{\partial x_{i}}=\frac{\partial \xi^{j}}{\partial x_{i}} \frac{\partial \varphi}{\partial \xi^{j}}=\frac{A_{i}^{j}}{J} \frac{\partial \varphi}{\partial \xi^{j}}
$$

Here $A_{i}^{j}$ is the Cartesian area component and $J$ is the Jacobian determinant defined by

$$
\vec{A}^{(k)}=A_{n}^{k} \vec{i}_{n}=\vec{e}_{(l)} \times \vec{e}_{(m)} \quad J=\vec{e}_{(1)} \cdot\left(\vec{e}_{(2)} \times \vec{e}_{(3)}\right) \quad \vec{e}_{(i)}=\frac{\partial x_{n}}{\partial \xi^{i}} \vec{i}_{n}
$$

and $k, l$ and $m$ are cyclic. The values of $\delta \xi^{i}$ are free to be chosen. In the present work $\delta \xi^{i}$ is chosen equal to unity and hence:

$$
\vec{A}^{(k)}=\delta \vec{A}^{(k)} \quad J=\delta V
$$

Here $\delta \vec{A}^{(k)}$ and $\delta V$ are the surface area vector and the volume of the control volume, respectively. The superscript $k$ indicates that the surface area vector describes a surface with a normal vector in $k$-direction.

\section{Discretised equations and power law scheme}

The coordinate transformations produce

$$
\hat{J}_{n n}^{i}=\left(\rho \hat{U}^{i} \varphi-\Gamma G^{i j} \frac{\partial \varphi}{\partial \xi^{j}}\right)_{n n}
$$

where

$$
\hat{U}^{i}=\vec{U} \cdot \vec{A}^{(i)}=u_{j} A_{j}^{i} \quad G^{i j}=\frac{\vec{A}^{(i)} \cdot \vec{A}^{(j)}}{J}=\frac{A_{k}^{i} A_{k}^{j}}{J}
$$

When the coordinate system is orthogonal, $G^{i j}$ is equal to zero for $i \neq j$. Then the total flux through a given face is divided into orthogonal and non-orthogonal parts, $\hat{J}_{o}^{i}$ and $\hat{J}_{N O}^{i}$

$$
\hat{J}_{n n}^{i}=\left(\hat{J}_{O}^{i}\right)_{n n}+\left(\hat{J}_{N O}^{i}\right)_{n n}
$$

where

$$
\hat{J}_{o}^{i}=\rho \hat{U}^{i} \varphi-\Gamma G^{i i} \frac{\partial \varphi}{\partial \xi^{i}} \quad \hat{J}_{N O}^{i}=-\left(\Gamma G^{i j} \frac{\partial \varphi}{\partial \xi^{j}}\right)_{i \neq j}
$$

After the above transformations, an integrated conservation equation written by curvilinear non-orthogonal coordinates appears. This equation contains a convective term, an orthogonal diffusive term and a non-orthogonal diffusive term.

The orthogonal flux, $\hat{J}_{o}^{i}$, is discretised in the same manner as on regular grids (e.g. Cartesian), for instance by the power law scheme. In the power law scheme, the two neighboring points (one upstream and one downstream) are used when the flux at the surface is calculated. When the convective term is much higher than the diffusive term (high Peclet number), only the information from the upstream point is transported to the surface. This scheme is second order accurate at low Peclet number but first order at high Peclet numbers. Following the methodology of Patankar (1980) and Karki (1986), the orthogonal flux for the power law scheme can be written as

$$
\left(\hat{J}_{o}^{1}\right)_{e}=A\left(P_{e}^{1}\right) D_{e}^{1}\left(\varphi_{P}-\varphi_{E}\right)+F_{e}^{1} \varphi_{P}
$$




$$
\left(\hat{J}_{O}^{1}\right)_{w}=A\left(-P_{w}^{1}\right) D_{w}^{1}\left(\varphi_{W}-\varphi_{P}\right)+F_{w}^{1} \varphi_{P}
$$

where

$$
A\left(P^{i}\right)=\left|\left[0,\left(1-0.1\left|P^{i}\right|\right)^{5}\right]+\right|\left[0,-P^{i}\right]
$$

and

$$
P_{n n}^{i}=\frac{F_{n n}^{i}}{D_{n n}^{i}} \quad F_{n n}^{i}=\left(\rho \hat{U}^{i}\right)_{n n} \quad D_{n n}^{i}=\left(\frac{\Gamma G_{i i}}{\delta \xi^{i}}\right)_{n n}
$$

Where $\mid[]$ means the largest of the expressions contained within it.

For the non-orthogonal diffusive terms, a linear variation of the dependent variables, together with central differencing, is used. If the non-orthogonal terms are discretised implicitly, both positive and negative coefficients appear. However, when an iterative method is used for the solution of the discretised equations, it is preferable to work with positive coefficients, since negative coefficients may produce numerical instability and unrealistic solutions. To achieve this, the non-orthogonal flux, $\hat{J}_{N o}^{i}$, is lumped into the source term, and then the non-orthogonal part of the equations is treated explicitly. Here, $\bar{S}_{2 P}$ has to be selected negative to ensure a numerically stable solution. The final equations to be solved have the form

$$
a_{P} \varphi_{P}=\sum_{n b} a_{n b} \varphi_{n b}+b
$$

where $n b$ indicates summation over neighboring grid points and

$$
a_{P}=\sum_{n b} a_{n b}-\bar{S}_{2 P} \quad b=b_{N O}+\bar{S}_{1 P}
$$

where

$$
b_{N O}=\left[\Gamma G^{12} \frac{\partial \varphi}{\partial \xi^{2}}\right]_{w}^{e}+\left[\Gamma G^{21} \frac{\partial \varphi}{\partial \xi^{2}}\right]_{s}^{n}
$$

and the coefficients in the $\xi$-direction for the power law scheme are

$$
a_{E}=D_{e}^{1} A\left(P_{e}^{1}\right) \quad a_{W}=D_{w}^{1} A\left(-P_{w}^{1}\right)
$$

with similar coefficients $a_{n b}$ in the $\eta$-direction. It has now been shown how the power law scheme is implemented. In a similar way the first order upwind scheme can be included (Patankar, 1980; Versteeg and Malalasekera, 1995).

\section{The QUICK scheme}

In the present work, a higher order scheme is also tested. The scheme selected is called the QUICK scheme and the full name is quadratic upstream interpolation for convective kinetics (Fluent, 1996; Versteeg and Malalasekera, 1995). This scheme computes the face value of an unknown variable based on the values stored at the two upsteam cell centres and on a third cell centre at the adjacent downstream point. This scheme can produce under- or overshoots. In the QUICK scheme used by Fluent, limiters are included to bound the solution. The QUICK scheme computes the face value with second or third order accuracy, depending upon the manner the limiters and boundedness are implemented and used. 


\section{Near wall region}

Several methods for modelling the turbulence in the near wall region are presented in the literature, and two methods are tested in this work. These two methods deal with the near wall turbulence in quite different ways. The first method is called Lawof-the-wall, and in this approach the viscous layer is jumped over by the use of a simple equation. This reduces the number of the grid points in the wall region. If the grid is too fine, the wall adjacent cell will be inside the viscous layer, and the method will not be recommended. The other method resolves the viscous and the buffer layer, and a fine grid near the wall is hence needed. This method is called the Two-layer zonal model. Some important aspects for the two different methods will briefly be discussed. The differences between the two methods are discussed with a basis in the $k-\varepsilon$ model.

The Law-of-the-wall assumes a fully turbulent flow, and requires that the walladjacent cell is in the turbulent region. For the grid this implies a relatively coarse grid near the wall. Near the wall the following equation is used

$$
\frac{U_{P} C_{\mu}^{1 / 4} k_{P}^{1 / 2}}{(\tau / \rho)_{w}}=\frac{1}{\kappa} \ln \left(E y_{P} \frac{C_{\mu}^{1 / 4} k_{P}^{1 / 2}}{v}\right)
$$

where $\kappa$ is von Karmans constant (0.42), $E$ is an empirical constant (9.81), $U_{p}$ is the mean velocity at point $P, C_{\mu}$ is an empirical constant $(0.09), k_{P}$ is the turbulent kinetic energy at point $P, \rho$ is density, subscript $w$ means wall, $y_{P}$ is the distance from the wall to point $P$. This is, as stated above, only valid in the turbulent region, and if the first grid point is to close to the wall, another equation is used. For

$$
E y_{p} \frac{C_{\mu}^{1 / 4} k_{p}^{1 / 2}}{v}<11.225
$$

the equation used is

$$
\frac{U_{P} C_{\mu}^{1 / 4} k_{P}^{1 / 2}}{(\tau / \rho)_{w}}=y_{P} \frac{C_{\mu}^{1 / 4} k_{P}^{1 / 2}}{v}
$$

These two last equations are often written like $u^{*}=(1 / \kappa) \ln \left(E y^{*}\right)$ and $u^{*}=y^{*}$.

The transport equation for $k$ is solved throughout the whole domain, with a zero gradient as a boundary condition at the wall. The source terms in the $k$-equation, the production of $k$ and its dissipation rate, have special treatments in the walladjacent cells. The $\varepsilon$-equation is not solved in the wall-adjacent cells. In the walladjacent cell the $\varepsilon$ is found from

$$
\varepsilon_{P}=\frac{C_{\mu}^{3 / 4} k_{P}^{3 / 2}}{\kappa y_{P}}
$$

For the Two-layer zonal model the viscous layer is discretised, and fully resolved. In the viscosity affected region, a length scale equation for the $\mu_{t}$ is implied. To separate the viscosity affected region from the fully turbulent region, a wall-distance based Reynolds number is used

$$
\operatorname{Re}_{y}=\frac{\rho \sqrt{k} y}{\mu}
$$


Two-layer Zonal model grid

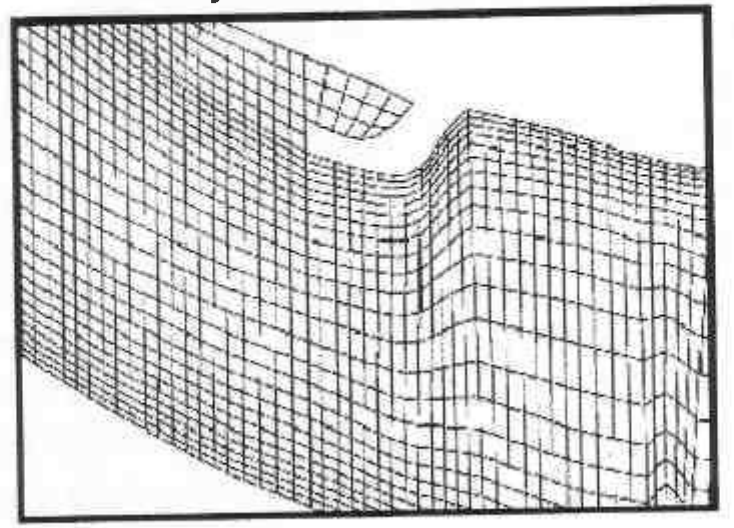

Law-of-the-wall grid

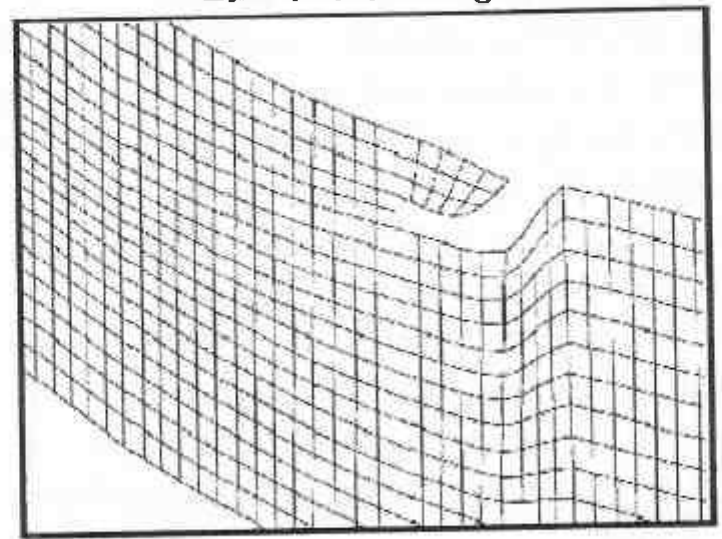

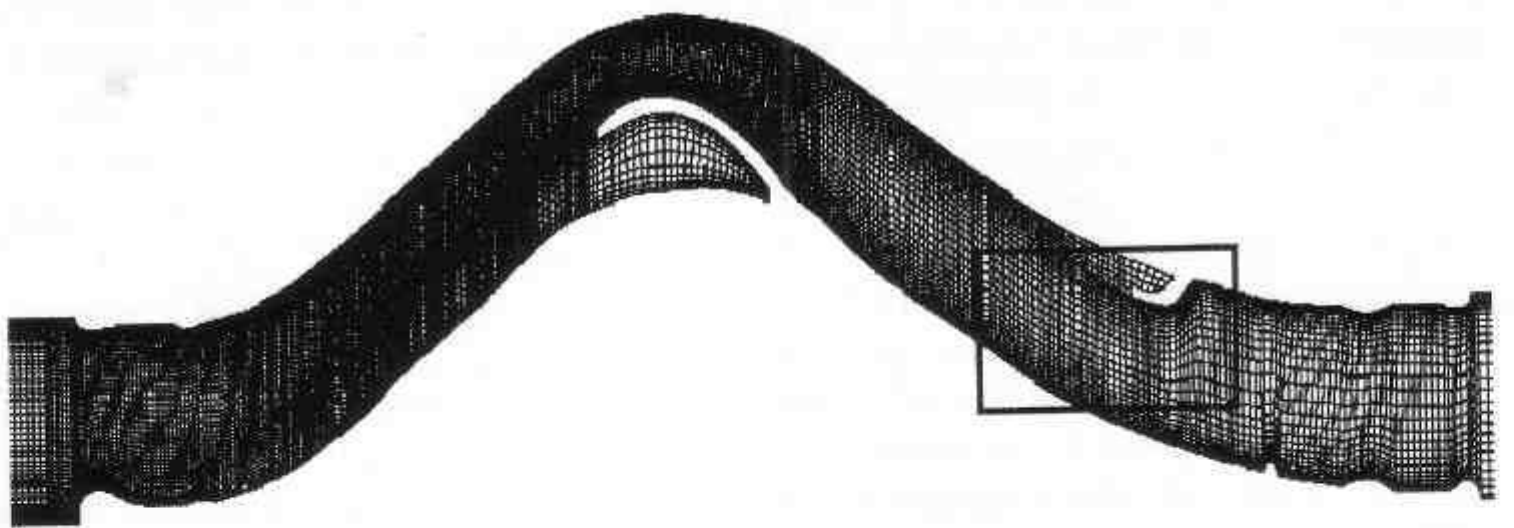

Figure 4. Structured grid.

For $\mathrm{Re}_{y}>200$ fully turbulent region is assumed. For $\operatorname{Re}_{y}<200$ viscous flow is assumed. When viscous flow is present, the turbulent viscosity is found through the use of

$$
\mu_{t}=\rho C_{\mu} \sqrt{k} \ell_{\mu}
$$

The $\varepsilon$-field is computed from

$$
\varepsilon=\frac{k^{3 / 2}}{\ell_{\varepsilon}}
$$

The length scales are found from

$$
\ell_{\mu}=c_{1} y\left[1-e^{\left(\mathrm{Re}_{y} / A_{\mu}\right)}\right], \quad \ell_{\varepsilon}=c_{1} y\left[1-e^{\left(\mathrm{Re}_{y} / A_{\varepsilon}\right)}\right]
$$

where $c_{1}$ is equal to $\kappa C_{\mu}^{-3 / 4}, A_{\mu}$ is equal to 70 , and $A_{\varepsilon}$ is equal to $2 c_{1}$.

The grid considerations are then opposite as it was for the Law-of-the-wall, and a relatively fine grid is required. This is accomplished for both grid-types, structured and unstructured. For the unstructured grid an adaptive method is used in order to achieve the wanted resolution. The Two-layer zonal model is not valid for the RSM model (Reynolds Stress Model), and hence is only used when the $k$ - $\varepsilon$ model is selected.

\section{Grids}

The grids selected are shown in Figure 4 and 5. The Law-of-the-wall grid is coarse by the wall. The number of cells is considerable higher for the simulations of the 
Two-layer Zonal model grid

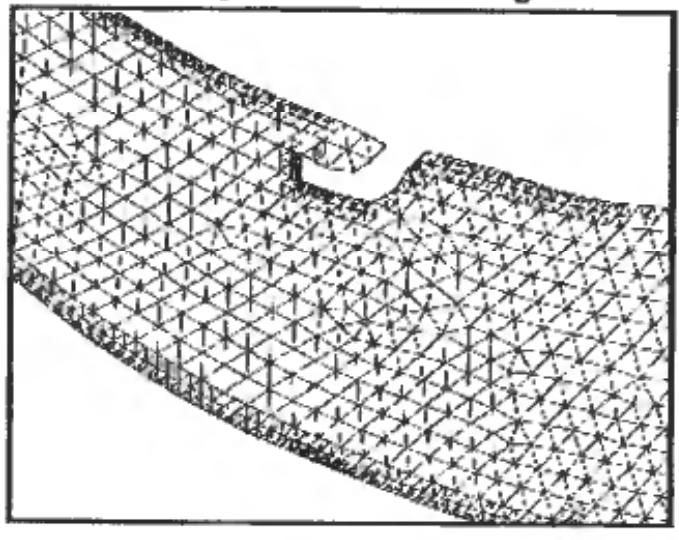

Law-of-the-wall grid

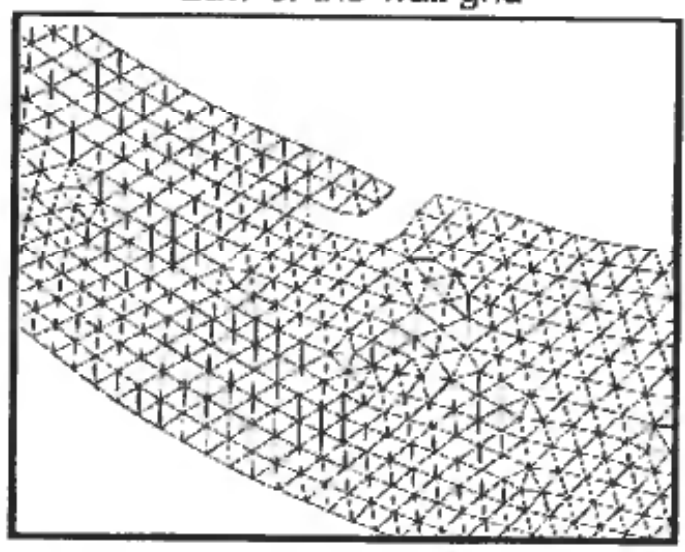

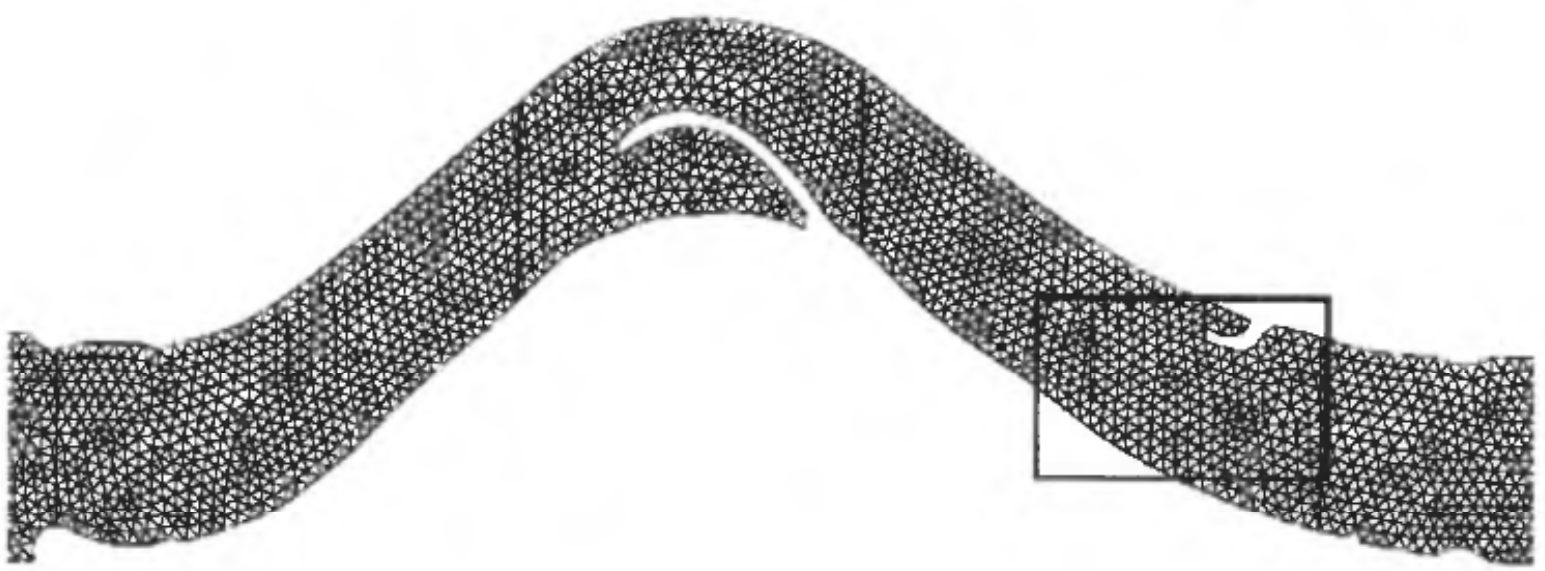

Figure 5. Unstructured grid.

Two-layer zonal model in comparison with the Law-of-the-wall since a fine grid is needed by the wall. For the structured simulations (Figure 4 ) the grid size was $875 \times 32(28000)$ cells for the Law-of-the-wall case, and $1440 \times 52(74880)$ cells for the Two-layer zonal model. For the unstructured simulations (Figure 5) the Law-ofthe-wall grid consisted of 30202 cells, and for the Two-layer zonal model the unstructured grid consisted of 52528 cells. For the unstructured cases, only triangle cells are used.

\section{Results}

The tested turbulence models are the standard $k-\varepsilon$ model and the RSM model. The standard $k-\varepsilon$ model is simple, robust, with known weakness. Anyway, it is still widely used in the CFD branch, mostly thanks to its overall properties, and considerably cheap computational cost. The RSM model is much more computational expensive, but offers unique knowledge about the different Reynold stresses in the flow. In the RSM model, the different Reynold stresses are individually solved by the use of differential equations. Fluent v4.5 is used when the grid is structured while Fluent v5.3 is used for the unstructured grid. Results from both first order and higher order schemes are shown. While QUICK is the higher order scheme for both Fluent $\mathrm{v} 4.5$ and v5.3, the first order scheme selected in these two codes are different. In Fluent v4.5 the power law scheme is used, while the first order upwind is used in Fluent v5.3. These schemes are close to equal. The SIMPLE algorithm is used to get a good coupling between the velocity and pressure fields. 
The analysed case has an inlet velocity equal to $3.54 \mathrm{~m} / \mathrm{s}$, and the turbulence intensity is set equal to $5 \%$. The simulation started $1 \mathrm{~m}$ upstream of the vane, and the velocity profile is close to fully developed when the flow reaches the vane. The inlet boundary conditions for the turbulence are therefore not important for the simulations of the vane. The flow properties of air at $20^{\circ} \mathrm{C}$ are used.

\section{Pressure drop}

Before presenting any comparisons between measured and predicted velocities, the pressure drops are studied (Table 3). The measured pressure drop is equal to 40.0 $\mathrm{Pa}$. The calculated pressure drops have large variation, from $35.0 \mathrm{~Pa}$ to $61.5 \mathrm{~Pa}$. The results from the structured grid have less variation compared to the unstructured grid. The two different wall treatments give different pressure drops, but no clear trends are seen. The pressure drops calculated by the $k$ - $\varepsilon$ turbulence model have variation from $-12.5 \%$ to $10.8 \%$, and the higher order scheme does not produce better results than the first order scheme. The RSM model overpredicts the pressure drop compared to both measurements and the $k-\varepsilon$ turbulence model, and the unstructured result is worst, especially when the unstructured grid is used together with the higher order scheme. The most likely reason for the increased predicted pressure drop for the RSM cases, compared to the other cases, is the overprediction of the recirculation zones and overprediction of the maximum velocities within the separator (seen from later figures).

Table 3. Pressure drops over the vane separator

\begin{tabular}{lcc}
\hline & \multicolumn{2}{c}{$\mathrm{DP}[\mathrm{Pa}]$} \\
\cline { 2 - 3 } Case & $\begin{array}{c}\text { First order } \\
\text { scheme }\end{array}$ & $\begin{array}{c}\text { Higher order } \\
\text { scheme }\end{array}$ \\
\hline Experimental & \multicolumn{2}{c}{40.0} \\
Structured, $k-\varepsilon$, Law-of-the-wall & 43.1 & 35.0 \\
Structured, $k$ - $\varepsilon$, Two-layer zonal model & 38.0 & 36.4 \\
Structured, RSM, Law-of-the-wall & 48.7 & 45.2 \\
Unstructured, $k-\varepsilon$, Law-of-the-wall & 44.3 & 42.8 \\
Unstructured, $k-\varepsilon$, Two-layer zonal model & 43.9 & 36.9 \\
Unstructured, RSM, Law-of-the-wall & 50.7 & 61.5 \\
\hline
\end{tabular}

\section{Mean velocities}

Comparisons between measured and predicted mean velocities will be presented from selected positions. The first profile to be presented is from the fully developed flow $50 \mathrm{~mm}$ upstream of the vanes. In an earlier work (Jøsang and Melaaen, 2000), it has been shown that it is important to have a fine grid by the wall when the Twolayer zonal model is used and similar a coarser grid by the wall when the Law-ofthe-wall model is used. A mismatch reduces strongly the quality of the solution in the fully developed region of the flow before the vanes. The presented results are based on the correct combination of grid and wall treatment. In Figure 6 the fully developed flow before the vane is seen. The left figure shows the results based on the structured grid, while the right has the unstructured grid results. For both types of 


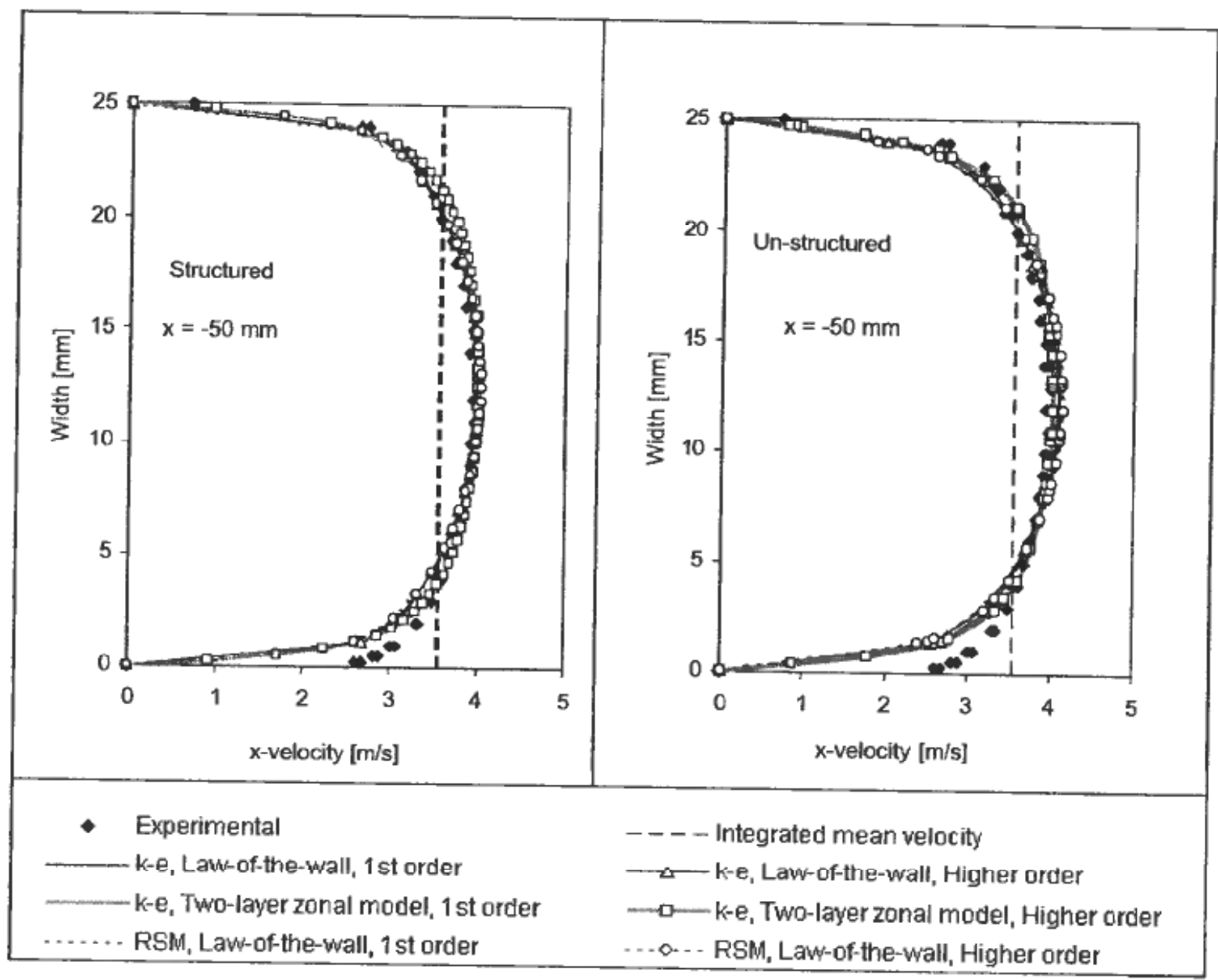

Figure 6. $\mathrm{x}$-velocity profiles at $\mathrm{x}=-50 \mathrm{~mm}$

grids six simulations have been done. Four of the simulations use the $k-\varepsilon$ turbulence model. The two different types of wall treatment are tested both together with the first order scheme and the higher order scheme. In addition, two simulations with the RSM model are shown. Both RSM simulations use the Law-of-the-wall model, but the first order and the higher order schemes are tested. The results in Figure 6 show that all the six simulations for the structured and unstructured grids fall together and it is good agreement with measurements. In the region near the bottom wall, there are some deviation between measurement and simulations. It is believed that this is due to inaccuracies in the measurements since the measurements are not symmetric.

The figures presented next are all for the $\mathrm{x}$-velocity components. The positions for the presented profiles are seen in Figure 2. The first results inside the vane separator $(x=50 \mathrm{~mm})$ are shown in Figure 7. The distance between the walls is different from $25 \mathrm{~mm}$ since the wall thickness changes from position to position. This figure shows that the simulated velocity profiles are close to equal, but are some higher than measured in the central part of the flow. In this part of the vane no separation appears and the flow field seems simple. The next profile is taken in the region over the hook (Figure 8). In this region the air is accelerated and a satisfactory agreement between measurement and simulations is shown, even though the simulations give the highest velocities.

The measured velocities in $\mathrm{x}=87.5 \mathrm{~mm}$ show a strong recirculation zone, extending almost $5 \mathrm{~mm}$ from the wall, with backflow velocities about $2 \mathrm{~m} / \mathrm{s}$ (Figure 9). 


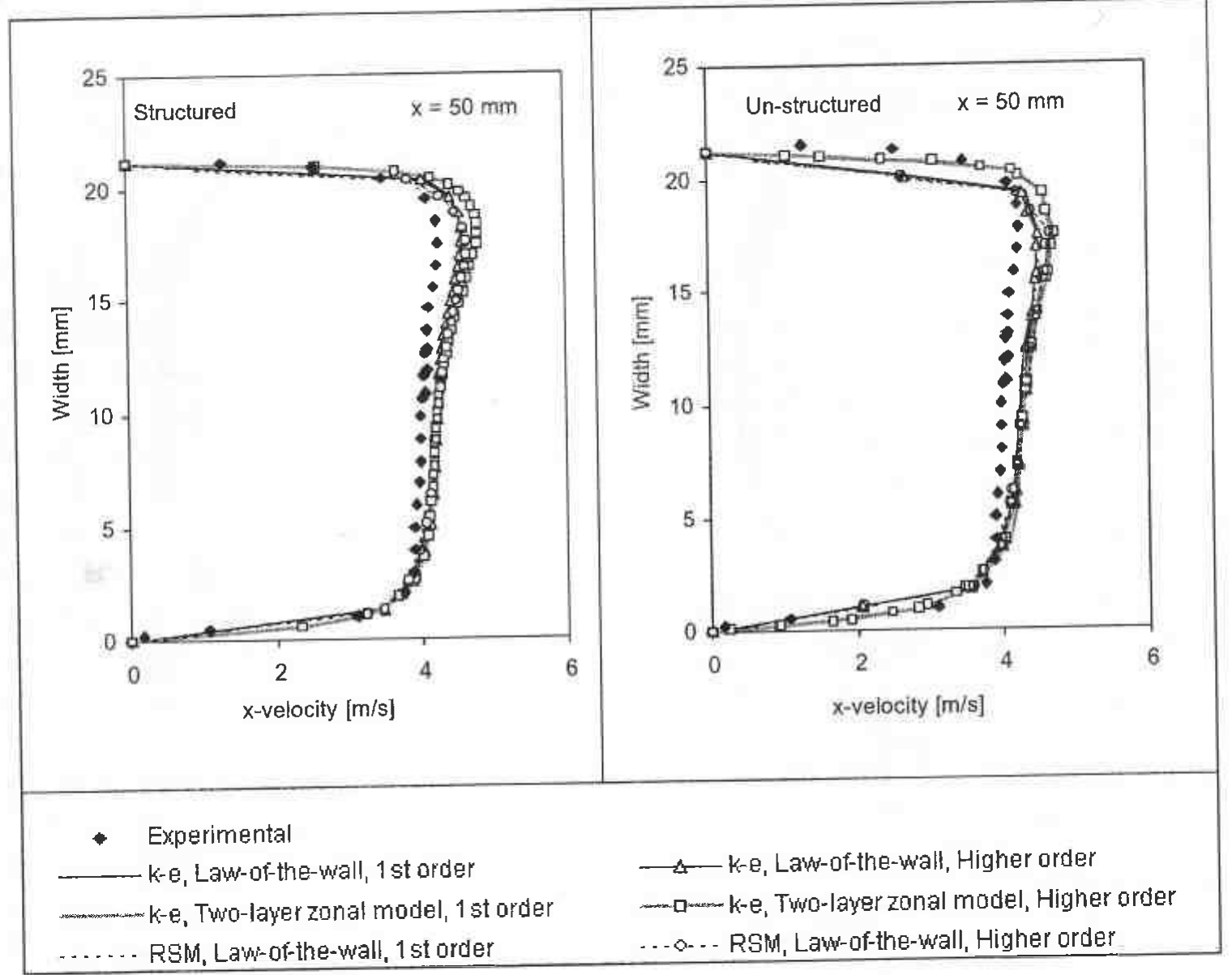

Figure 7. $\mathrm{x}$-velocity profiles at $\mathrm{x}=50 \mathrm{~mm}$.

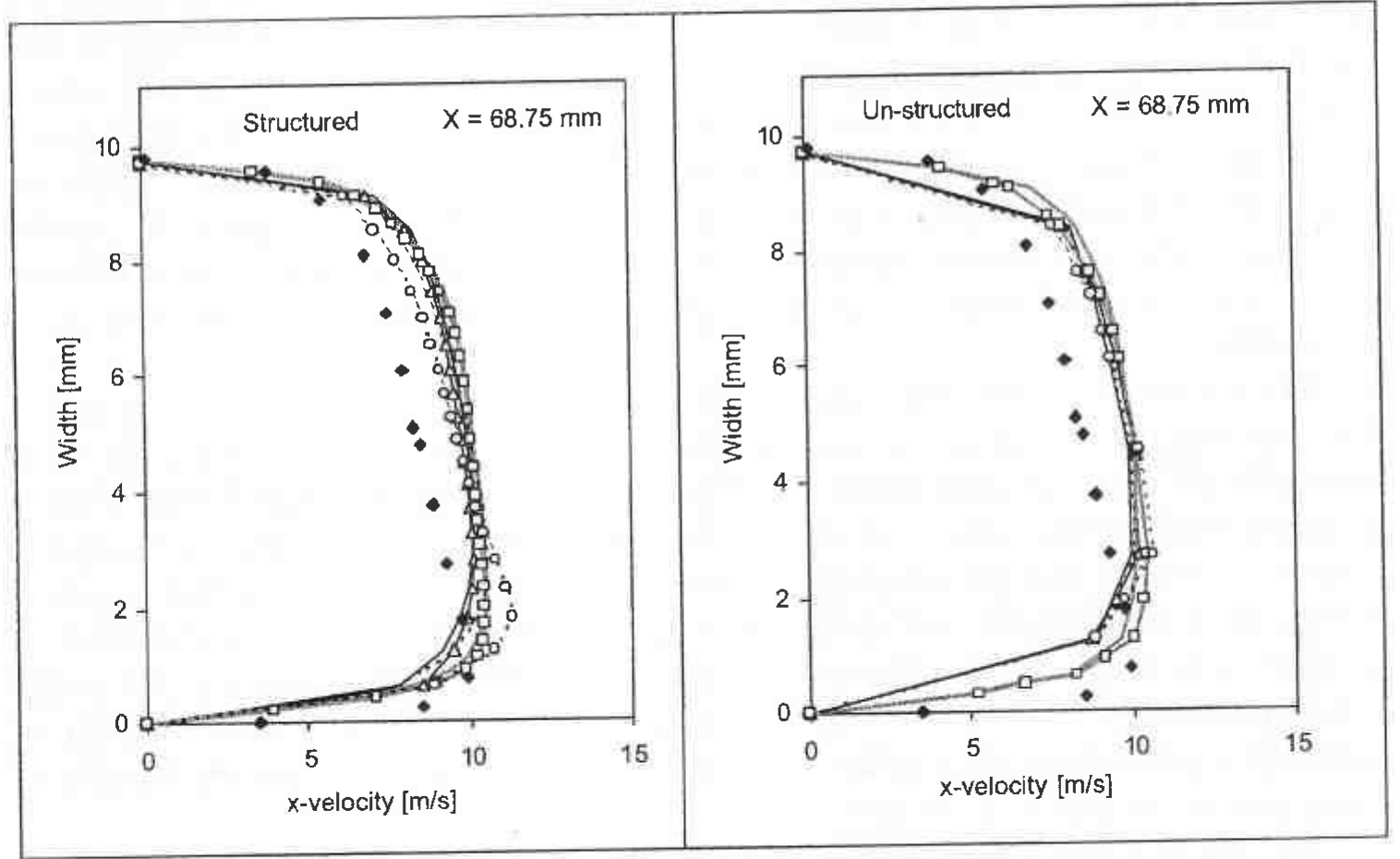

Figure 8. $\mathrm{x}$-velocity profiles at $\mathrm{x}=68.75 \mathrm{~mm}$ (legend in Figure 7). 


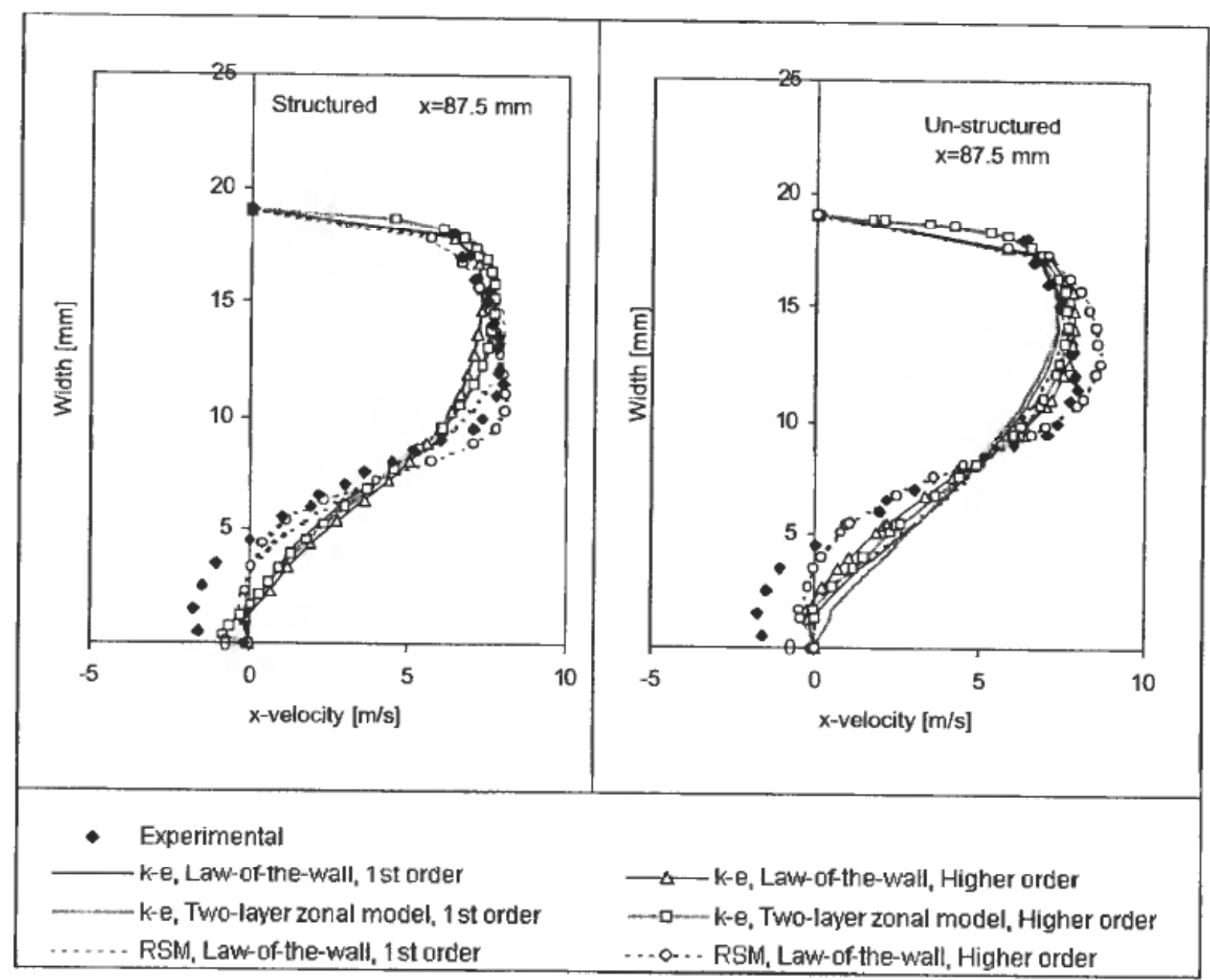

Figure 9. $x$-velocity profiles at $x=87.5 \mathrm{~mm}$.

Again, the structured cases are shown to the left, and the unstructured cases to the right. The profiles of the RSM model are closest to the experimental values. The recirculation zone is however not so good predicted. The transition from negative to positive $\mathrm{x}$-velocity is well predicted, even though the magnitude of the negative velocities is not correct predicted. The $k-\varepsilon$ model predicts somewhat linear velocity profile, ending in a negative velocity close to the wall. For the unstructured cases the deviations are similar as for the structured cases, but for the RSM model the first and higher order schemes produce different results.

The next profile presented is the profile located at $x=112.5 \mathrm{~mm}$ (Figure 10) after the small hook. The measured values imply that the recirculation zone is not present. This profile shows that the RSM case has not forgot the recirculation zone in the same degree as the experimental values shown. The structured RSM case is closer to the experimental values, while the unstructured RSM case nearly predicts a recirculation zone and has a much to high maximum velocity. This overprediction leads probably to an increase in the prediction of the pressure drop. The $k-\varepsilon$ cases perform well, with some overprediction near the lower wall.

For the profiles at $\mathrm{x}=137.5 \mathrm{~mm}$ a recirculation zone is present at the upper wall. Figure 11 shows the structured and unstructured cases for this position. The predicted values in position $\mathrm{x}=137.5 \mathrm{~mm}$ coincide well with measurements for most of the $k-\varepsilon$ cases except in the recirculation zone. The RSM case overpredicts the positive velocity, and misses the magnitude of the backflow. The shape of the profile in the 


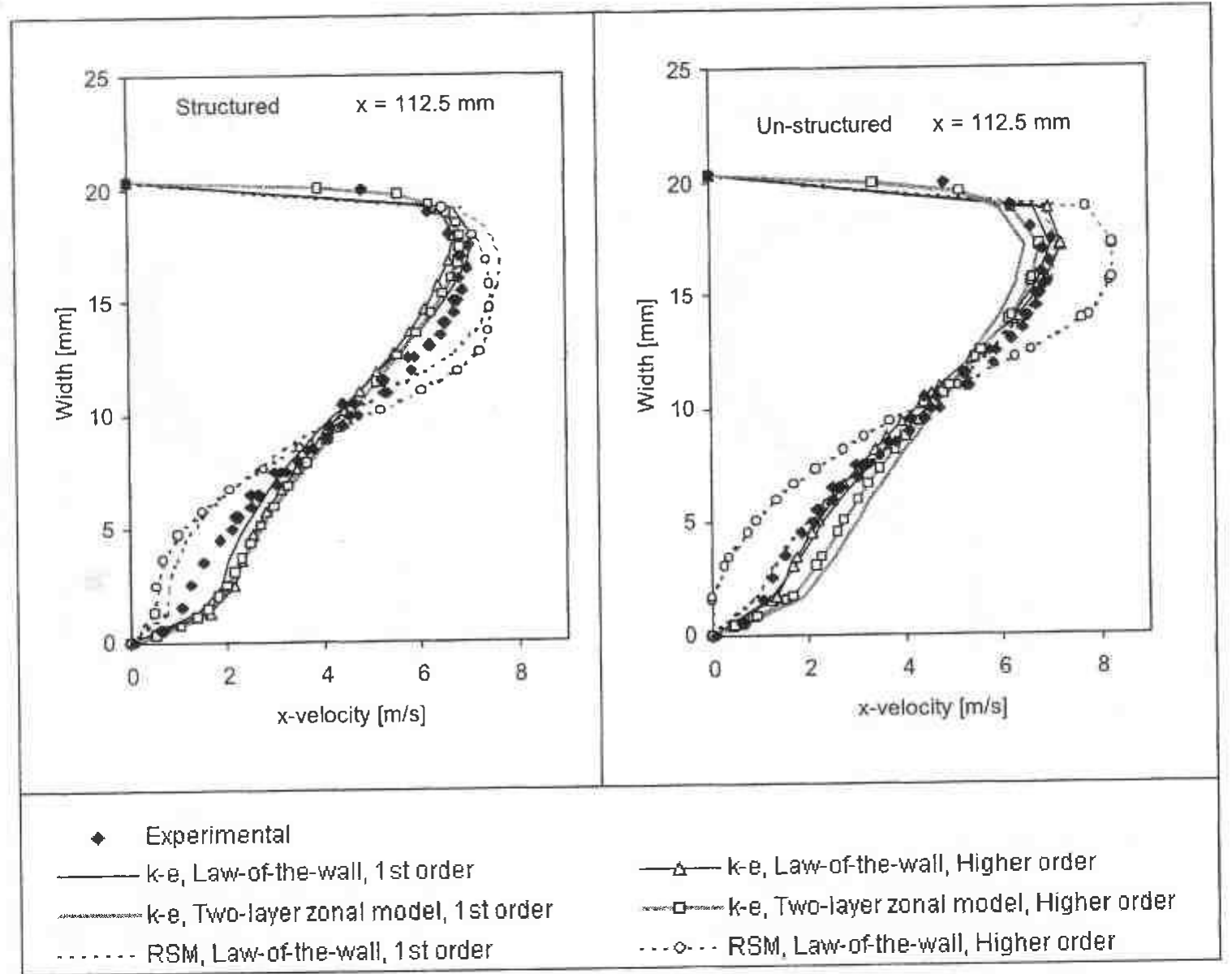

Figure 10. $\mathrm{x}$-velocity profiles at $\mathrm{x}=112.5 \mathrm{~mm}$.

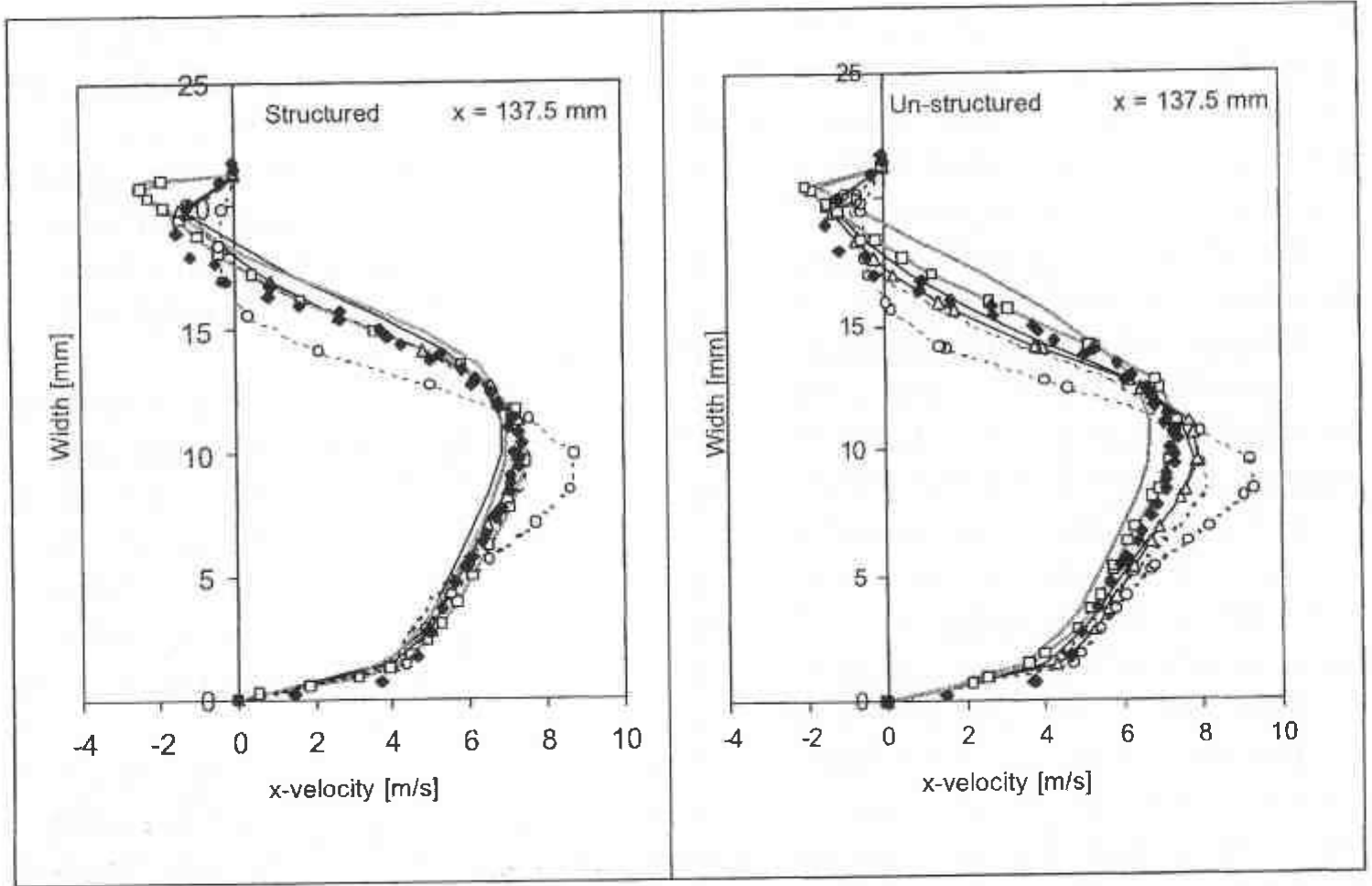

Figure 11. $\mathrm{x}$-velocity profiles at $\mathrm{x}=137.5 \mathrm{~mm}$ (legend in figure 10 ). 


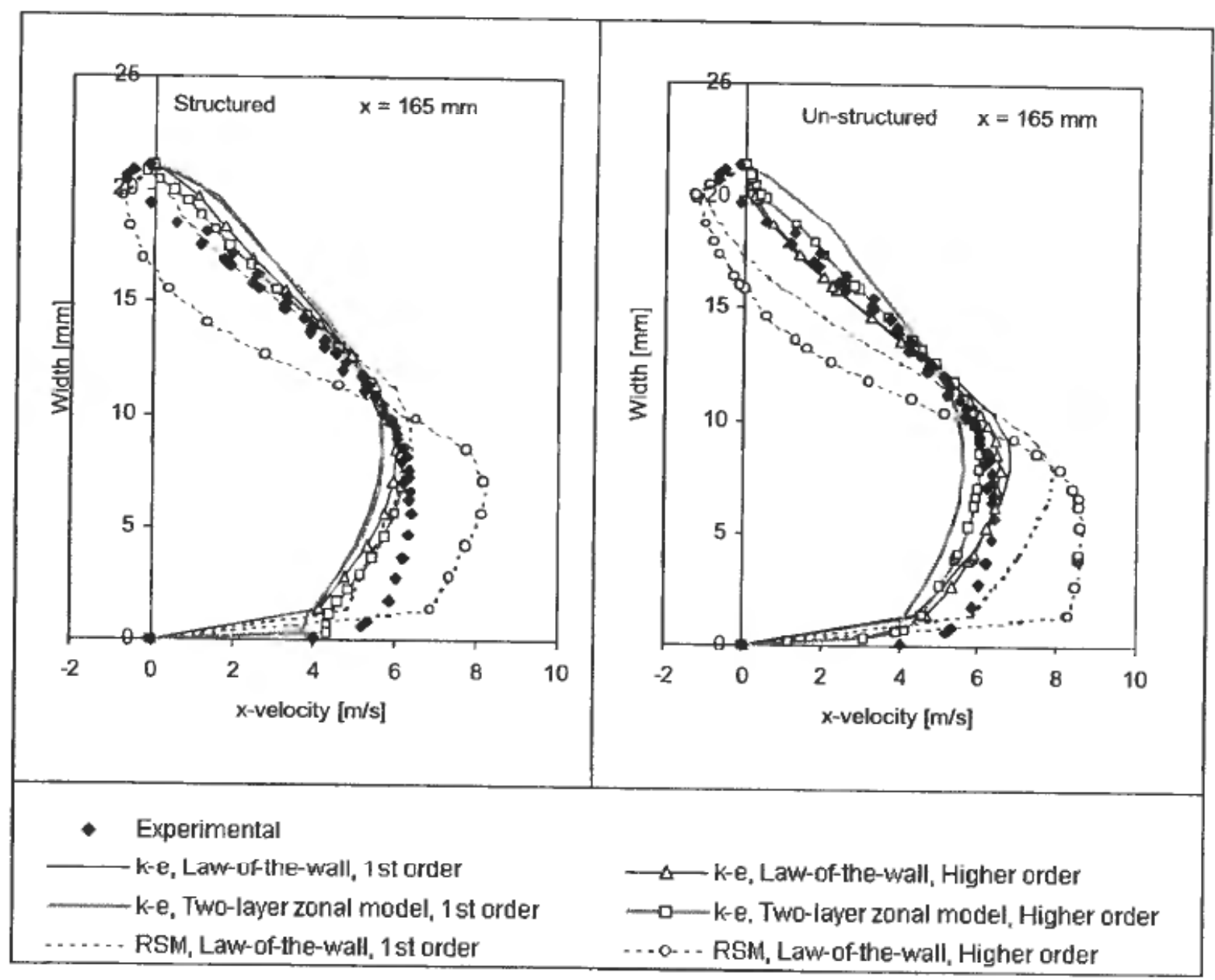

Figure 12. $\mathrm{x}$-velocity profiles at $\mathrm{x}=165 \mathrm{~mm}$.

backflow area is better for the RSM model. The $k-\varepsilon$ cases are somewhat steeper towards the upper wall.

At $\mathrm{x}=165 \mathrm{~mm}$ (Figure 12) some backflow is measured in few points and with low magnitude. The RSM model overpredicts the positive $\mathrm{x}$-velocity and the backflow. This overprediction will probably lead to an increase in the predicted pressure drop. The $k-\varepsilon$ cases are satisfactory, but do not manage the small recirculation. Based on the different $x$-velocity profiles shown, there are no large difference between the two different wall treatments, the Law-of-the-wall and the Two-layer zonal model. The main difference is between the $k-\varepsilon$ turbulence model and the RSM model. The difference is most visible when the RSM model is used together with the higher order scheme. Overall the structured and unstructured results are similar, but for the combination of the RSM model and the higher order scheme, the unstructured model seems to produce the worst result, which is also in accordance with Table 3.

\section{RMS-velocities}

For the $\mathrm{x}$-rms velocities (velocity fluctuations due to turbulence) similar figures as shown for the $\mathrm{x}$-velocities will be presented. The $\mathrm{x}$-rms velocities are presented in Figure 13 for $\mathrm{x}=-50 \mathrm{~mm}$, and contains profiles from both the $k-\varepsilon$ model and the RSM model, the structured cases to the left and the unstructured cases to the right. For the $k-\varepsilon$ model the $\mathrm{x}$-rms velocity is derived from the turbulent kinetic energy. This is done by assuming that the fluctuations are equal in all three directions 


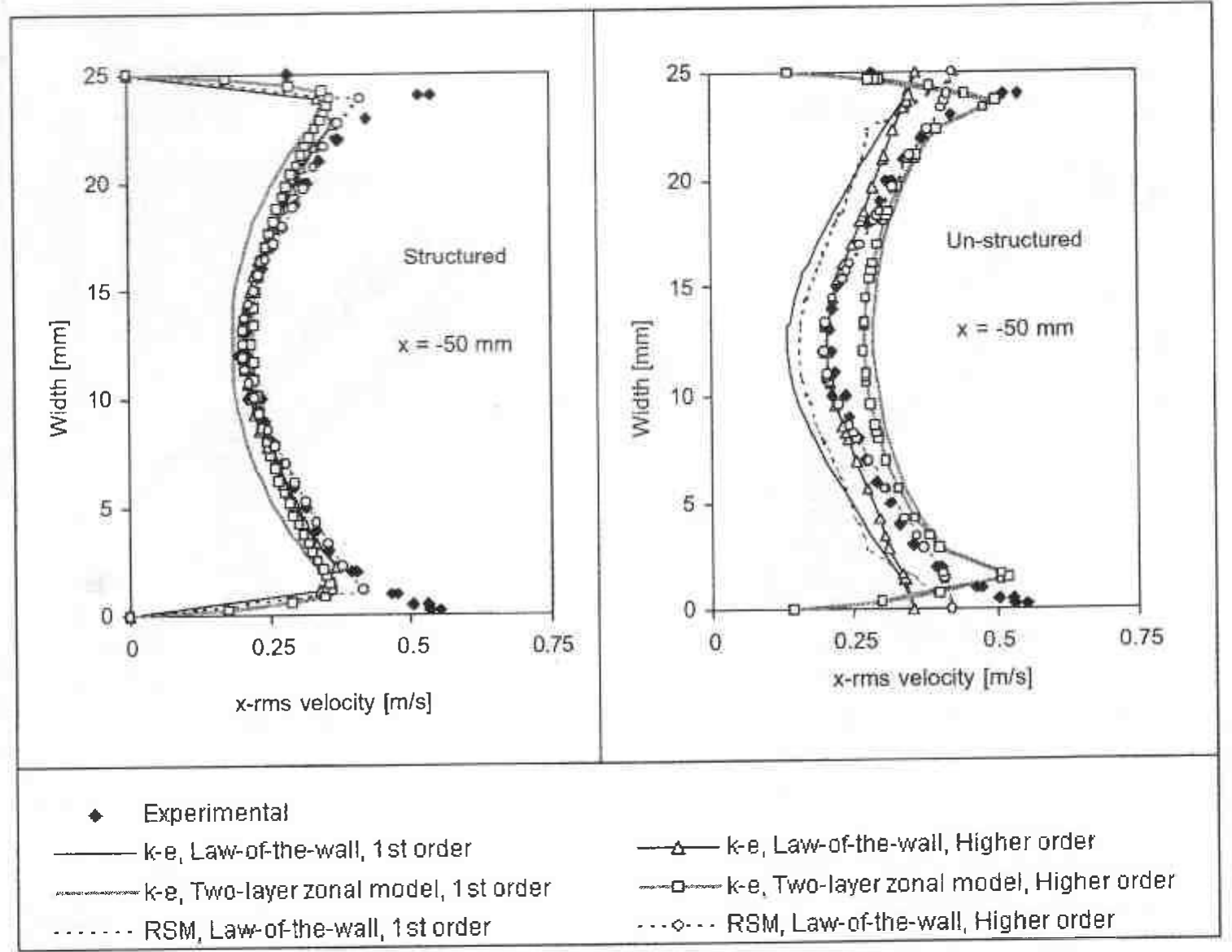

Figure $13 . \mathrm{x}-\mathrm{rms}$ velocity profiles at $\mathrm{x}=-50 \mathrm{~mm}$.

$\left(u^{\prime}=(2 k / 3)^{1 / 2}\right)$. It can be seen from Figure 13 that for the structured grid it is good accordance between the simulations, but the simulation based on the $k-\varepsilon$ model together with the Two-layer zonal model and first order scheme deviates some from the other simulations. The RSM model predicts very well. For the unstructured grid it is larger variation between the different simulations, but the RSM model together with the higher order scheme gives the best result.

The Figures 14 to 19 show the $\mathrm{x}$-rms velocities inside the vane separator. These fluctuation velocities are harder to simulate than the mean velocities, and hence the deviations between simulations and measurements are larger. It seems to be some larger deviation between the simulation results when the unstructured grid is used compared with the structured grid. It is not possible to draw a final conclusion about these results, but roughly the RSM model seems to predict the X-rms velocity better than the $k$ - $\varepsilon$ turbulence model. In some of the measured profile positions it is small deviation between the first order and the higher order simulation, while also large deviation is observed. The two different wall treatments produce results with some deviation, but it is not clear which is the best.

\section{Conclusion}

The air flow in a vane type separator is studied in this work. The simulations cover two different turbulence models and different numerical schemes. The selected turbulence models are the $k-\varepsilon$ turbulence model and the Reynolds Stresss Model (RSM). The simulations are done on both a structured and an unstructured grid. 


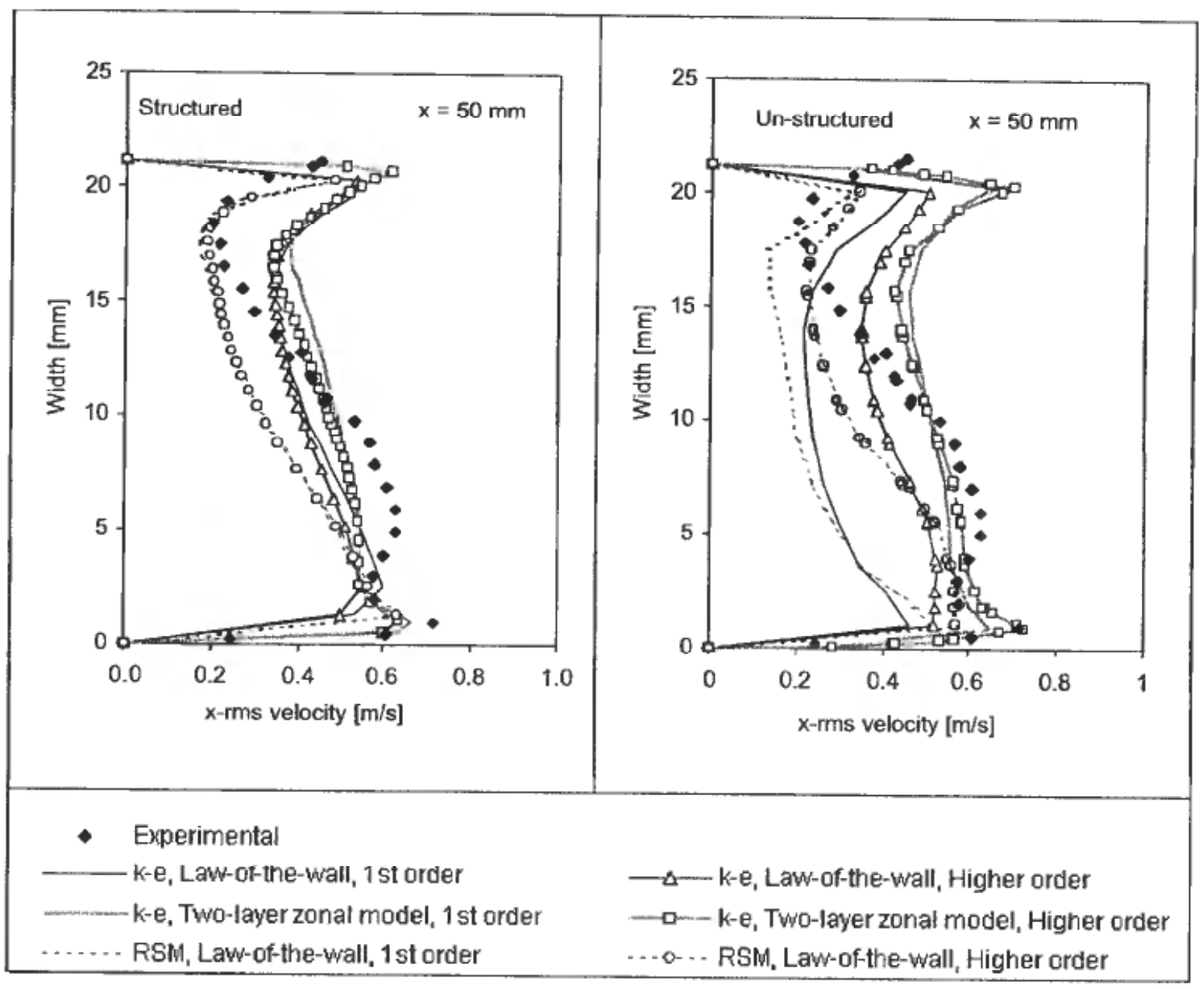

Figure 14. $\mathrm{x}$-rms velocity profiles at $\mathrm{x}=50 \mathrm{~mm}$.

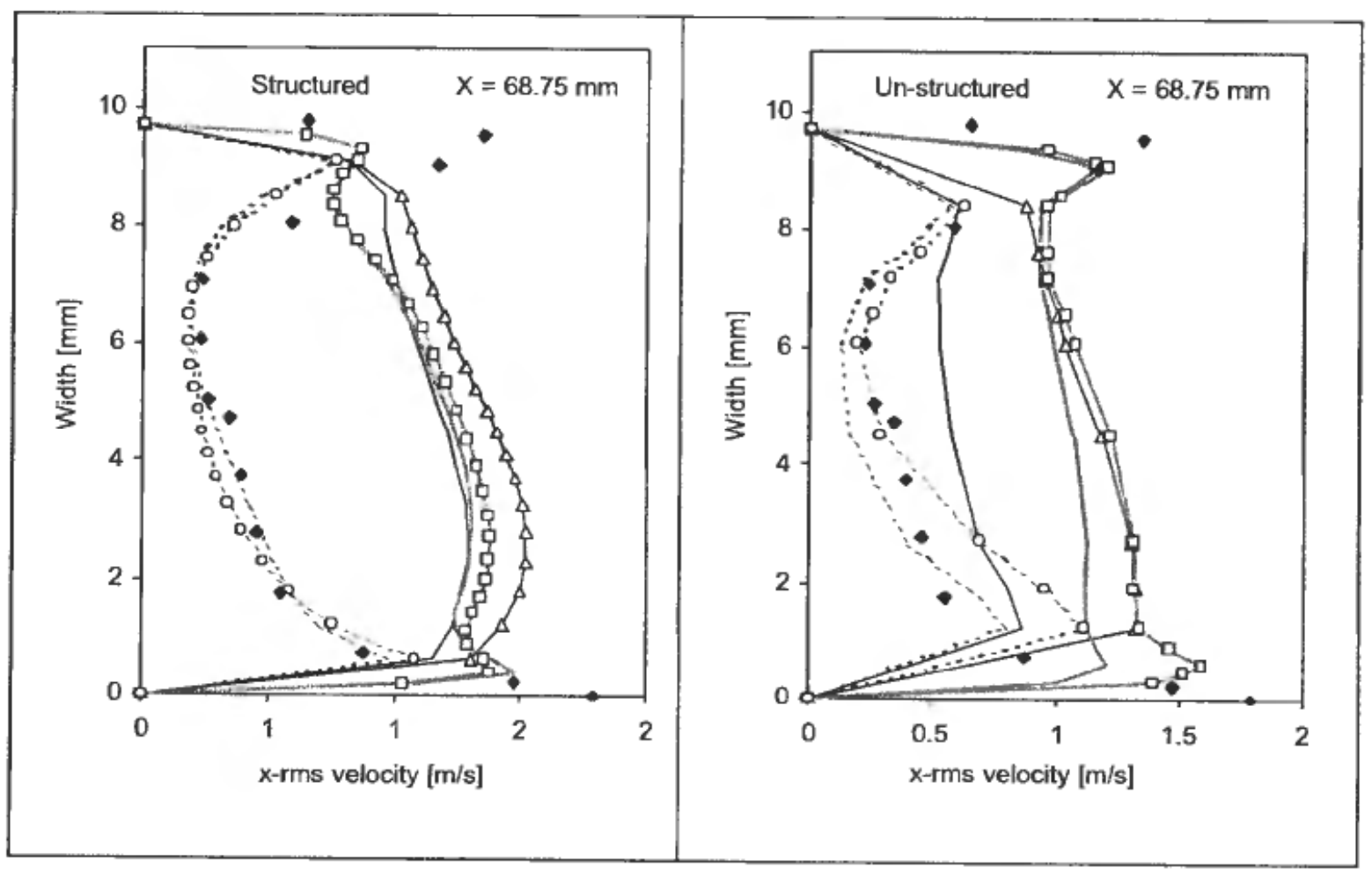

Figure 15. $x$-rms velocity profiles at $x=68.75 \mathrm{~mm}$ (legend in figure 14). 


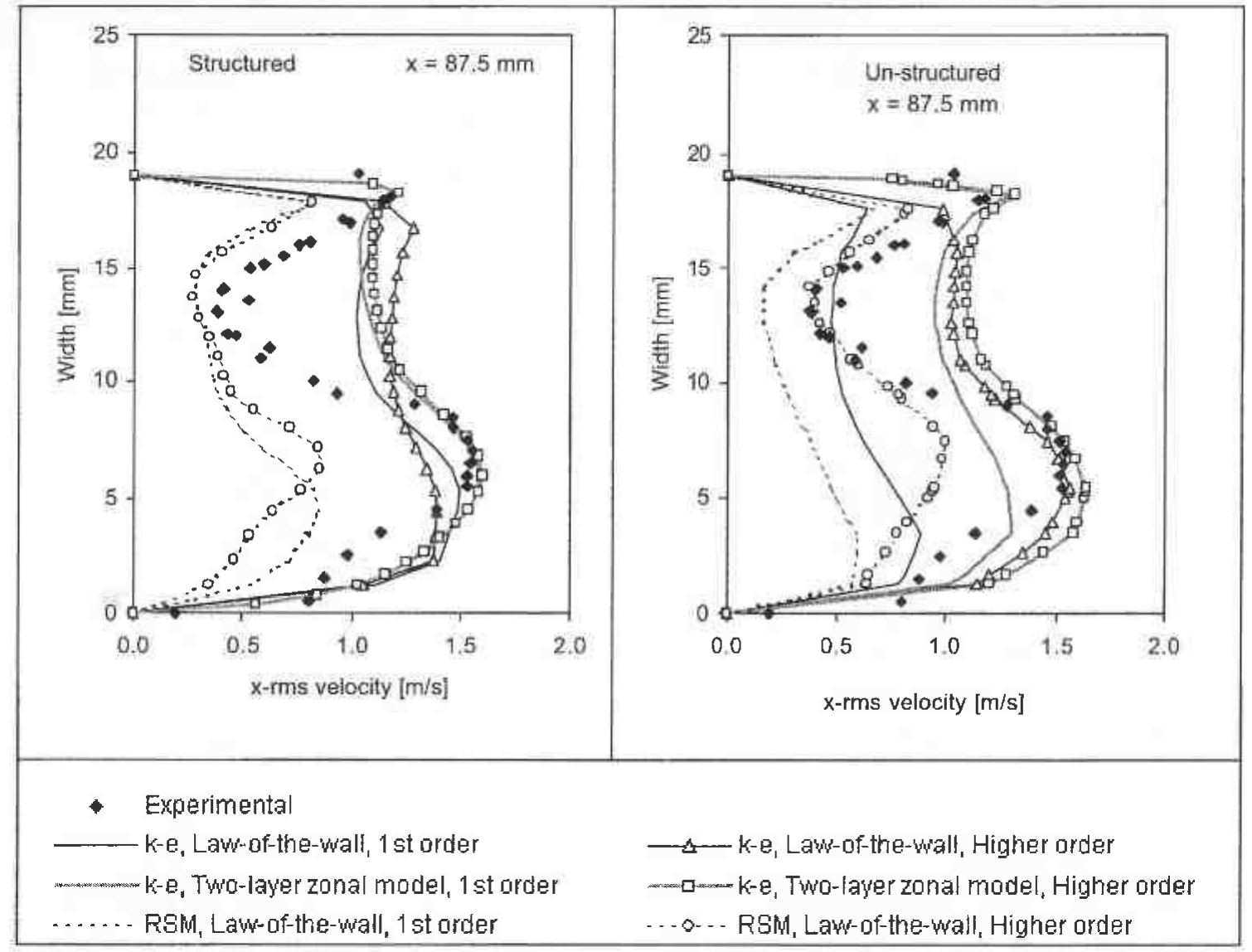

Figure 16. $\mathrm{x}$-rms velocity profiles at $\mathrm{x}=87.5 \mathrm{~mm}$.

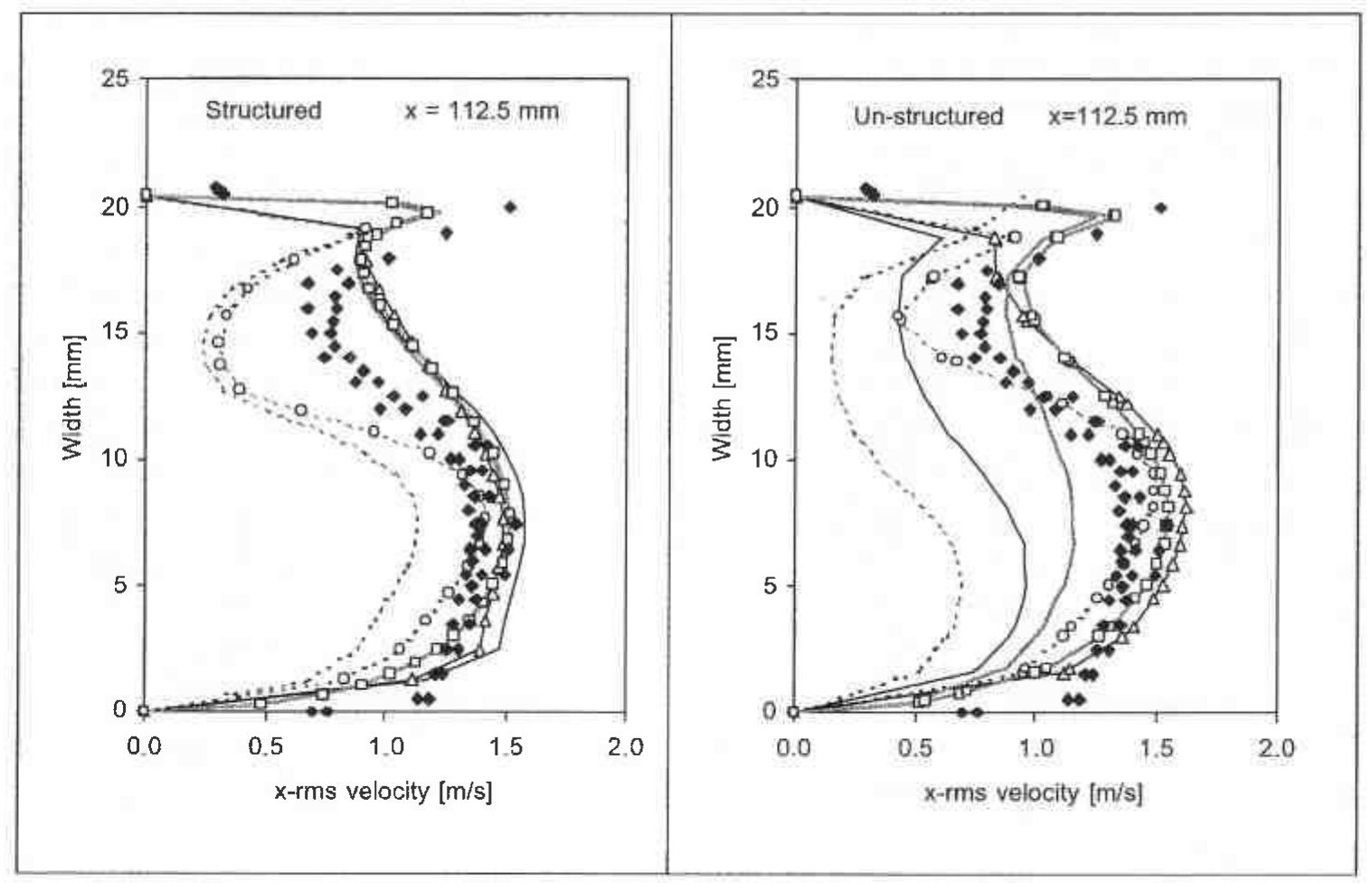

Figure 17. $\mathrm{x}$-rms velocity profiles at $\mathrm{x}=112.5 \mathrm{~mm}$ (legend in figure 16). 


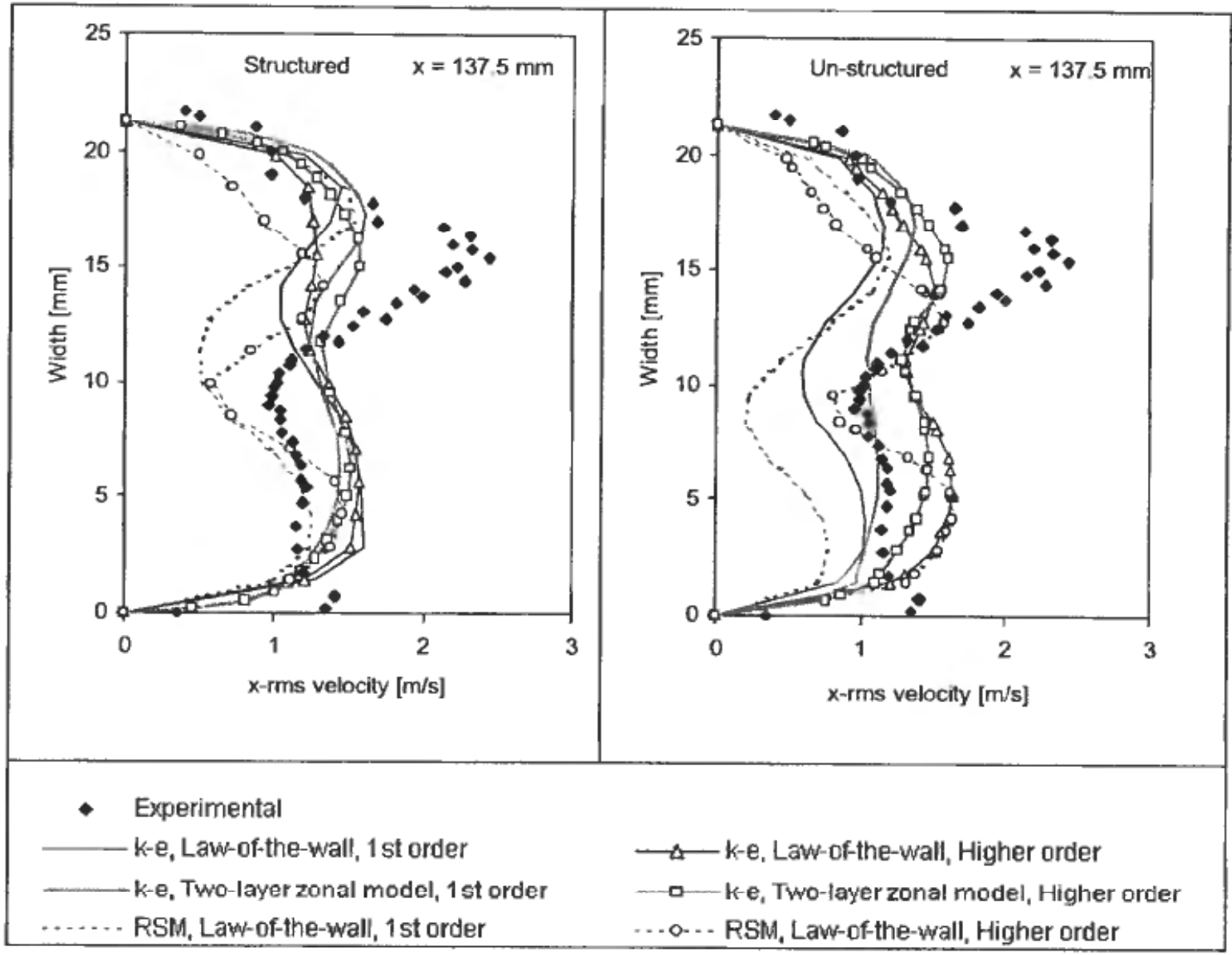

Figure 18. $\mathrm{x}$-rms velocity profiles at $\mathrm{x}=137.5 \mathrm{~mm}$.

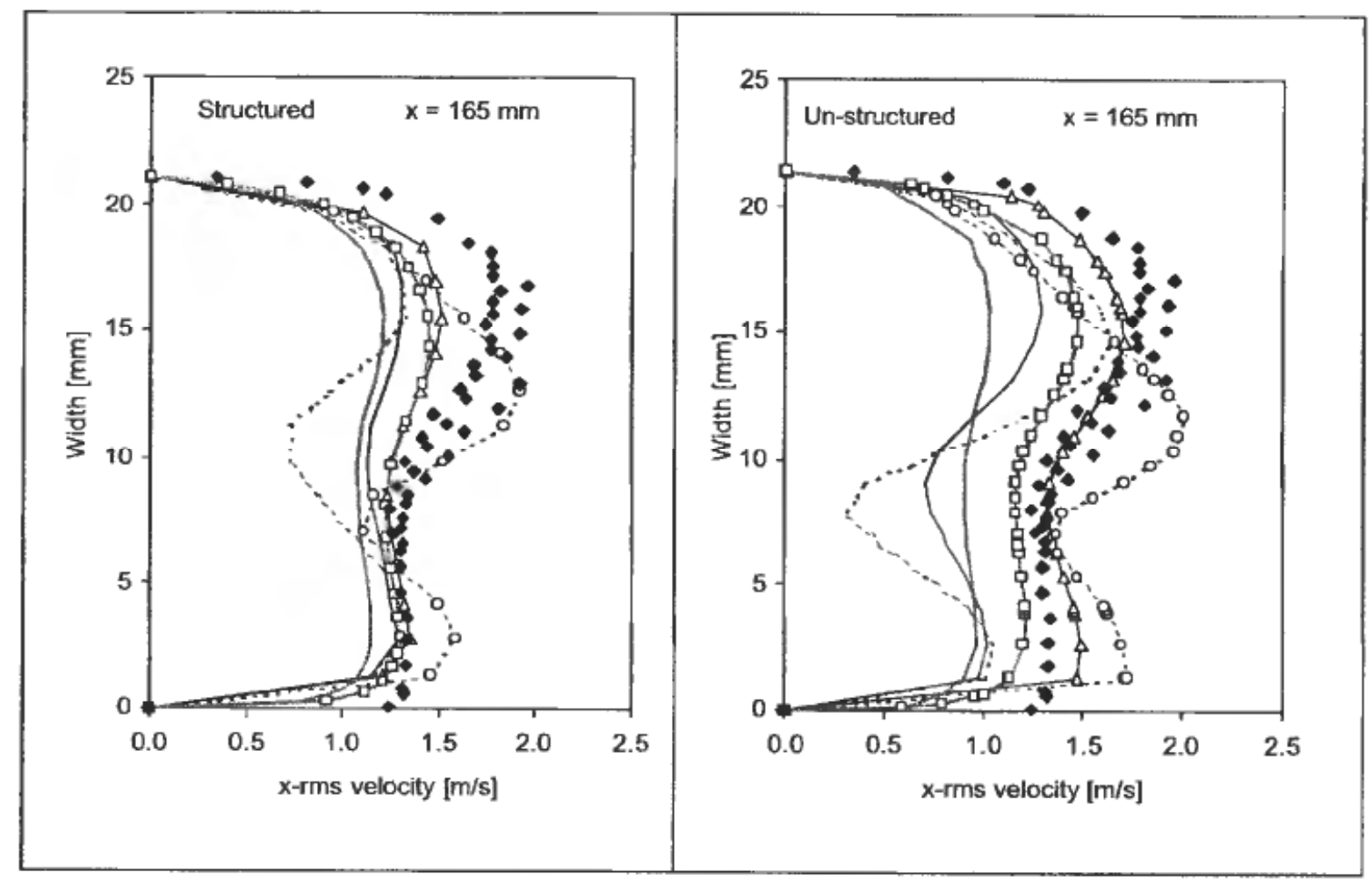

Figure 19. $\mathrm{x}$-rms velocity profiles at $\mathrm{x}=165 \mathrm{~mm}$ (legend in figure 18). 
The selected numerical schemes are the first order scheme (Fluent v5.3 uses the first order upwind and Fluent v4.5 uses the power law scheme), and the higher order QUICK scheme. Also two different wall treatments are included in this analysis.

When the flow enters the separator, the flow is influenced both by pressure gradients, curvature and obstacles, and the quality of the predictions varies. In the backflow regions the predictions are somewhat insufficient. The width, how far the recirculation zone extends into the passage and the magnitude of the backflow is not always satisfactorily. The predicted pressure drop is closely related to the prediction of the recirculation zones within the separator.

In the comparison between the simulations and measurements, the pressure drop, the mean velocities and the fluctuation velocities ( $\mathrm{rms}$ velocities) are analysed. The different combination of models produce different results, and it is not easy to have a final conclusion. The differences between the structured and unstructured simulations are usually small. Normally, the highest order scheme is believed to produce the most accurate results, but in the present work the combination of the best turbulence model (RSM) and QUICK on the unstructured grid gave the worst result. The pressure drop was overpredicted by $50 \%$. The pressure drops are predicted around $\pm 13 \%$ for the $k-\varepsilon$ turbulence model. The $k-\varepsilon$ turbulence model combinations gave satisfactory predicted flow fields, although some deviations exist for the prediction of the recirculation zones. To differentiate between the $k-\varepsilon$ and the RSM models is not straight forward, since the quality of the predictions varies from position to position in the separator, and depends on which variable is analysed (pressure drop, mean velocity or rms velocity). For this vane separator a first order scheme together with the $k-\varepsilon$ turbulence model seems best.

\section{REFERENCES}

Fluent USER's Guide, Release 4.4, August 1996.

Jøsang, Aa. I. and MelaAen, M. C. (2000). Detailed measurements and simulations of a vane separator. International Conference on Applied Computational Fluid Dynamics, October 17-20, Beijing, China, 2000.

KARKI, K. C. (1986). A calculation procedure for viscous flows at all speeds in complex geometries, Ph.D. thesis, University of Minnesota.

Keshava, Iyer V., Dasgupta, A., Venkat, Rao B. and Srivastava, R. (1997). Some investigation on flow through chevrons of moisture separator and reheater used in nuclear power plants, Proceedings of the ASME Fluids Engineering Division, FED-Vol. 244, ASME 1997, pp. 381-386.

Kudo, Y., Tanaka, N. and Miyano, H. (1996). An analytical study on the moisture separation performance in dryers, International Conference on Nuclear Engineering, Volume 1Part A, ASME 1996, pp. 123-129.

Monat, J. P., McNulty, J., Michelson, I. S. and Hansen, O. V. (1986). Accurate evaluation of chevron mist eliminators, Chemical engineering progress, December 1986.

NakaO, T., Nagase, M., Aoyama, G. and Murase, M. (1999). Development of simplified wave-type vane in BWR steam dryer and assessment of vane droplet removal characteristics, Journal of Nuclear Science and Technology, Vol. 36, No. 5, May 1999, pp. 424432.

Patankar, S. V. (1980). Numerical heat transfer and fluid flow, Hemisphere, Washington, D.C.

VerlaAn, C. (1991). Performance of novel mist eliminators, Ph.D. thesis, Faculty of Mechanical Engineering and Marine Technology, Delft University of Technology.

Versteeg, H. K. and Malalasekera, W. (1995). An Introduction to Computational Fluid Dynamics - the Finite Volume Method. Longman, England.

WANG, W. and Davies, G. A. (1996). CFD studies of separation of mists from gases using vane-type separators, Trans. IChemE, Vol. 74, Part A, March 1996, pp. 232-238.

WANG, Y. and JAMES, P. W. (1998). The calculation of wave-plate demister efficiencies using numerical simulation of the flow field and droplet motion, Third International Conference on Multiphase Flow, ICMF'98, Lyon, France. 